\title{
Discrete-Time Approximations of the Holmström-Milgrom Brownian-Motion Model of Intertemporal Incentive Provision ${ }^{1}$
}

\author{
Martin Hellwig* \\ University of Mannheim \\ Klaus M. Schmidt** \\ University of Munich and CEPR
}

This version: May 5, 1998

\begin{abstract}
This paper studies the relation between multi-period discrete-time and continuous-time principal-agent models. We explicitly derive the continuous-time model as a limit of discrete-time models with ever shorter periods and show that the optimal incentive scheme in the continuous model, which is linear in accounts, can be approximated by a sequence of optimal incentive schemes in the discrete models. For a variant of the discrete-time model in which the principal observes only total profits at the end of the last period and where the agent can destroy profits unnoticed we show, that if the length of each period is sufficiently small, then an incentive scheme that is linear in total profits is approximately optimal.
\end{abstract}

KEYwords: Principal-agent problems, linear incentive schemes, intertemporal incentive provision, Brownian motion.

JEL CLASSifiCATion Numbers: C61, D82, J33.

\footnotetext{
${ }^{1}$ For helpful comments and discussions we are grateful to Darell Duffie, Oliver Hart, Bengt Holmström, Nobuhiro Kiyotaki, John Moore, Holger Müller, Sven Rady, and Jae Sung. We are also grateful for research support from the Schweizerischer Nationalfonds, the Deutsche Forschungsgemeinschaft, and the Taussig Chair at Harvard University.

*Department of Economics, University of Mannheim, D-68131 Mannheim, email: hellwig@pool.unimannheim.de

** Department of Economics, University of Munich, Ludwigstr. 28 (Rgb.), D-80539 Munich, Germany, email: klaus.schmidt@lrz.uni-muenchen.de
} 


\section{Introduction}

This paper studies the relation between multi-period discrete-time and continuous-time principal-agent models in the seminal paper by Holmström and Milgrom (1987). The purpose is to obtain a better understanding of the structural elements underlying the linearity of optimal incentive schemes in some continuous-time models. We share the view of Holmström and Milgrom that the nonlinearities or even discontinuities of optimal incentive schemes that are typical for static principal-agent models are unlikely to be robust to changes in the models, in particular, to changes that allow for manipulation of the information required to implement them. However, the argument presented by Holmström and Milgrom does not make the underlying structure entirely clear, at least to us.

The Holmström-Milgrom paper involves three different models:

1. A static model, in which the agent chooses a (finite) vector of probabilities over possible states of the world. This model is used to show that if the action space of the agent is sufficiently rich (of "full dimensionality" in the underlying probability space), then for any action in the interior of the action set, there exists at most one incentive scheme implementing this action.

2. A multi-period model, which is a $T$-fold repetition of the static model. It is shown that if the technology controlled by the agent is stationary and if the principal and the agent have utility functions with constant absolute risk aversion, then the optimal incentive scheme in the $T$-period model is a simple $T$-fold repetition of the optimal incentive scheme in the one-period model, with no attention paid to the order in which the different outcomes arise.

3. A continuous-time model in which the agent controls the drift rate vector (but not the variance) of a multi-dimensional Brownian motion. It is suggested that this model can be obtained as a limit of a sequence of multi-period discrete-time models specified in such a way that the overall duration of the principal-agent relation is fixed and, along the sequence of models, periods become shorter as well as more numerous. The different dimensions of the multi-dimensional Brownian motion 
correspond to the different states of the world or outcomes in the static model and the different "accounts" showing how often a given outcome arises in a multi-period model. As in the multi-period discrete-time models, the assumptions of constant absolute risk aversion and stationarity in the technology ensure that the optimal incentive scheme in the continuous-time model depends only on the cumulative change in this vector of "accounts", with no attention paid to the details of the underlying time paths.

As reported so far, the results of Holmstöm and Milgrom provide for "linearity" of optimal incentives in "accounts" listing the frequencies of incidence of different outcomes, but not necessarily for linearity in outcome variables such as total revenues and costs, or profits. This reflects the fact that "outcomes" in the Holmström-Milgrom analysis correspond to "states of the world" in the static model which are defined without any reference to variables such as revenues, costs, or profits that would permit aggregation across states or - in the intertemporal models - across accounts. Even if such outcome variables are introduced, it is not clear that incentives should rely on linear aggregates that treat, e.g., two profit realizations of one as equivalent to one profit realization of two and one profit realization of zero. This is obviously not an issue if the static model involves only two possible outcomes. In this case, aggregate profits can be written as a constant plus the number of times the high-profit outcome occurred times the difference between profit levels across outcomes, so trivially linearity in accounts and linearity in profits are equivalent. However with more than two outcomes, linearity in accounts and linearity in profits are no longer equivalent, and one needs additional assumptions to justify the use of incentive schemes that are linear in profits or in some other outcome aggregate.

Within their continuous-time model, Holmström and Milgrom give two assumptions which yield the linearity with respect to outcome variables that they are really after. One of these is the assumption that the agent's effort cost depends only on a linear aggregate of the drift rate vector that he controls. The other is the assumption that the principal observes only a linear aggregate of the different "accounts", i.e., the different dimensions of the Brownian motion whose drift rate vector the agent controls. Either assumption ensures that an optimal incentive scheme involves only the corresponding linear aggregate of the vector of cumulative changes in "accounts". If the weighting used in these linear 
aggregates reflects some underlying outcome variable so that e.g. an outcome involving the profit realization "two" twice as much weight as an outcome involving the profit realization "one", the incentive scheme is actually linear in this outcome variable.

Unfortunately, both these routes to obtaining linearity of optimal incentive schemes in outcome variables are given only for the continuous-time models. In either case it is not clear what analogues these results have in the multi-period discrete-time models that serve to approximate the Brownian motion model. In consequence it is difficult to disentangle the respective roles of intertemporal aggregation in the Brownian motion model and the additional assumptions in providing for the linearity of optimal incentive schemes.

The difficulty is partly due to the fact that Holmström and Milgrom are very sketchy about the relation between discrete-time and continuous-time models. To see that this is a nontrivial matter, note that in a discrete-time model the process indicating how many times each outcome has been observed up to time $t$ is nondecreasing in $t$, negative instances of observation being out of the question. To get such a process to converge to a Brownian motion, one must be looking at the "accounts" process relative to some norm so that a negative change can be interpreted as shortfalls of the actual frequency of a given outcome from the norm. The question then is where this norm comes from and how it is specified. Holmström and Milgrom do not say anything about this. Nor do they say anything about the specification of outcome variables and their dependence upon the periodization.

Our paper fills this gap. We link the one-period, the multi-period, and the continuoustime models of Holmström and Milgrom in a unified framework and explicitly derive the continuous-time model as a limit of discrete-time models with ever shorter periods. We will indicate the class of Brownian models that can be approximated this way.

Given our account of the precise relation between the multi-period discrete-time and the continuous-time models of Holmström and Milgrom, we look for discrete-time analogues of their linearity results for the continuous-time model. Two sets of results are obtained. First we show that there is no discrete-time analogue for the linearity result that is based on effort cost being a function of a linear aggregate of the drift vector that the agent controls. For this specification of effort costs, the corresponding discrete- 
time models involve isoquants that are straight lines in the space of vectors assigning probabilities to the different possible outcomes. Therefore, these models typically have boundary solutions assigning probability zero to all but very few outcomes. Indeed, in the absence of any further restrictions the discrete-time models will have solutions concentrating all probability mass on the two outcomes closest to the target value for mean returns. Thus, except for a gap left by the discretization of outcomes, all risk and hence all agency costs of an inefficient allocation of risk will disappear. If one looks at a sequence of such discrete-time models with ever shorter periods these boundary solutions do not converge to anything resembling the solution that Holmström and Milgrom derive for the Brownian motion model with this specification of effort costs.

Our second major result shows that there is a discrete-time analogue for the Holmström-Milgrom result that in the Brownian motion model a linear incentive scheme is optimal if the principal observes only a linear aggregate of the different dimensions of the Brownian motion whose drift vector the agent controls. To obtain this analogue, we assume that in discrete-time, the principal does not observe the time path of the outcome process at all. He only observes a final aggregate. Moreover, by the time he observes the aggregate, the agent may have manipulated it by destroying some returns that had actually been realized. With this assumption about asymmetric information concerning outcomes, we prove that the linear incentive scheme that is optimal in the continuous model is approximately optimal in the discrete-time models if the period length is sufficiently short. The argument relies on the continuity of certain features of the model in the transition between discrete and continuous time. Stationarity of solutions to discrete-time models, which is central for Holmström and Milgrom, plays no role here because under the given information assumption such stationarity typically is not obtained.

Our results link up with the basic intuition that Holmström and Milgrom had provided for their analysis. In motivating their paper they had argued that nonlinearities and even more so, discontinuities - in incentive schemes are vulnerable to manipulation by the agent. However much of their actual analysis does not involve this notion of manipulation at all. Manipulation enters their analysis only in the case where the principal observes only a linear aggregate of the different dimensions of the Brownian-motion process; it plays no role in their multi-period discrete-time analysis or in the other results 
they have for the Brownian motion model. Our results show that for their specification of multi-period discrete-time and continuous-time models, the linearity result that is based on asymmetric output observation and the scope for manipulation that this introduces is the only one that has a discrete-time analogue. Within the context of their analysis, concerns about manipulation must be the underlying force behind linearity.

This being said, we must however point to the companion paper by Hellwig (1998a), which does develop a linearity result based on the specification of the cost function, without any concern about manipulation. However, this result involves a specification in which variances and diffusion parameters are endogenous and effort cost depends on both, drift rates and diffusion parameters. This specification cannot be accommodated within the framework of Holmström and Milgrom which presumes exogenously given diffusion terms.

The Holmström-Milgrom paper has given rise to a large literature, but only a few papers are concerned with the methodological and mathematical underpinnings of the analysis. Most papers in the literature just appeal to their results to justify the use of linear incentive schemes in applications; for an example see Holmström and Milgrom (1991). Among the more method-oriented papers, Schättler and Sung (1993) develop a general mathematical framework for the study of agency problems when the agent controls the drift of a Brownian motion in continuous time; their results strengthen and greatly extend the continuous-time results of Holmström and Milgrom (1987). Sung (1995) further extends the analysis by allowing for moral hazard with respect to risk choices, more precisely, choices concerning the diffusion parameters of a Brownian motion. Sung (1997) develops the corresponding analysis for continuous-time agency problems concerning jump processes. Bolton and Harris (1997) also consider continuous-time problems concerning jump processes as well as diffusions. However they are concerned with first-best rather than second-best problems, considering optimal risk sharing and optimal actions without concern for incentive compatibility and showing that for arbitrary preference specifications risk-sharing considerations will typically call for nonlinear contracts in a first-best setting.

The above-mentioned papers all work directly in continuous time and do not discuss the relation between static or multi-period discrete-time and continuous-time agency models. Multi-period discrete-time agency models and their relation to continuous-time 
models are studied by Schättler and Sung (1997) and by Müller (1997). Unlike Holmström and Milgrom (1987) these papers consider multi-period models as $T$-fold repetitions of a static model in which the agent 's effort choice determines the mean of a normally distributed random variable. Müller (1997) shows that, as in Mirrlees (1974), in a model of this type, the principal's problem typically does not have a solution because a first-best allocation can be approximated (but not reached) by a sequence of incentive schemes using penalties for low outcome realizations to discourage shirking, the incidence of penalties becoming ever rarer and the penalties themselves becoming ever more severe as one goes along the sequence. ${ }^{1}$ The conclusion is independent of the number of periods $T$ or the "length of one period" $1 / T$ in the multi-period model, but when $T$ becomes large and $1 / T$ goes to zero, the requisite penalties become large and their incidence becomes ever rarer even if the shortfall of payoff expectations from first-best is kept fixed. In Schättler and Sung (1997), existence of a solution to the principal's problem in discrete time is also a problem. To get around it the authors impose a prior restriction on the class of incentive schemes they admit; with this restriction, they find that conditions for implementing a given strategy of the agent are similar in a $T$-period discrete-time and in a continuous-time Brownian-motion model. Neither paper asks how a continuous-time Brownian-motion model would be approximated by discrete-time models with finitely many possible outcomes in any one period or what is the relation between linearity of incentive payments in "accounts" and linearity of incentive payments in outcome aggregates, e.g. in profits.

The plan of the paper is as follows. The next section develops the framework for the analysis by introducing one-period and multi-period models and specifying the role of the "length of the period" as a parameter of these models. Section 3 considers a sequence of control paths in the discrete models and shows that if this sequence converges to a well defined control path in the continuous model, then the incentive schemes that implement the control paths in the discrete models converge to an incentive scheme that implements the limit path in the continuous model. Whereas this result takes the convergence behavior of control paths as given, Section 4 shows that this convergence behaviour is actually obtained for suitable subsequences of optimal control paths and incentive schemes. Section 5 deals with the case where the agent's cost function depends on expected profits only.

\footnotetext{
${ }^{1}$ In Holmström and Milgrom (1987), this difficulty was avoided by the assumption that the set of states of the world in the static model is finite.
} 
Section 6 considers the case where the principal observes an accounting aggregate, such as total profits, but not the time paths of individual accounts. There we show that Holmström and Milgrom's main result on linearity in aggregates can indeed be approximated by a series of appropriately designed discrete models. All formal proofs are relegated to the appendix. In particular, Appendix A gives the formal proofs of all results in the text. Certain supplementary are given in Appendices B and C.

\section{A Discrete Multi-Period Model}

We start with the static model. Suppose that there is one period of length 1 . At the beginning of the period the agent chooses an action which gives rise to a stochastic outcome $\tilde{\pi} \in\left\{\pi_{0}, \ldots, \pi_{N}\right\}$. The outcome $\pi_{i} \in I R$ is interpreted as a profit level. Following Holmström and Milgrom (1987) we assume that the agent chooses the probability distribution $p$ over possible profit levels directly at personal cost $c(p) \geq 0$. Thus, the agent's action is $p=\left(p_{0}, \ldots, p_{1}\right) \in P$ where $P$ is the $N$-dimensional simplex. Throughout the paper we assume the following:

Assumption 1 The effort cost $c(p)$ of an action $p \in P$ is given by a function

$c(\cdot)$ on $\mathbb{R}^{N+1}$ which is strictly convex as well as continuously differentiable on some open set that contains the interior of $P$.

The agent is assumed to have a constant coefficient of absolute risk aversion $r>0$. Given an incentive scheme associating the payment $s_{i}$ to the outcome $\pi_{i}$, he chooses action $p \in P$ so as to maximize his expected utility

$$
\Leftrightarrow \sum_{i=0}^{N} p_{i} e^{-r\left(s_{i}-c(p)\right)} .
$$

From the first-order conditions for this maximization problem, one easily finds that to implement an action $p \in P$ such that the certainty equivalent of the agent's utility is 0 , an incentive scheme $s=\left(s_{0}, \ldots, s_{N}\right)$ must satisfy:

$$
s_{i} \leq c(p) \Leftrightarrow \frac{1}{r} \ln \left(1 \Leftrightarrow r c_{i}+r \sum_{j=0}^{N} p_{j} c_{j}\right)
$$


for $i=0, \ldots, N$, with equality if $p_{i}>0$, where, as usual, $c_{i}$ refers to the partial derivative of the effort cost function with respect to $p_{i}$. The argument is a straightforward adaptation of Theorem 3 in Holmström and Milgrom to allow for the possibility of boundary solutions, i.e., of actions $p$ involving $p_{i}=0$ for some $i$. As indicated by (2), if $p_{i}=0$ for some $i$, the corresponding incentive payment $s_{i}$ is not uniquely determined by incentive compatibility considerations. However, we assume that for actions $p$ involving $p_{i}=0$ for some $i$, we may set the corresponding incentive payment $s_{i}$ so as to satisfy (2) with equality. ${ }^{2}$

The principal is assumed to be risk neutral. His payoff from implementing an action $p$ by an incentive scheme $s=\left\{s_{0}, \ldots, s_{N}\right\}$ is given by:

$$
\sum_{i=0}^{N} p_{i}\left(\pi_{i} \Leftrightarrow s_{i}\right)
$$

Consider now a multi-period version of this model in which the agent can change his action at discrete points in time. We want to keep the total length of the time interval (which is normalized to 1) fixed, however, and increase the number of periods within this interval. Suppose that there are $\frac{1}{\Delta}$ periods, each of length $\Delta \in\left\{1, \frac{1}{2}, \frac{1}{3}, \ldots\right\}$, which are indexed by $\tau=\left\{1, \ldots, \frac{1}{\Delta}\right\}$. In order to make the one-period problem and the $\frac{1}{\Delta}$-period problem comparable we have to reformulate the model:

In each period there are $N+1$ profit levels $\pi_{i}^{\Delta}, i \in\{0, \ldots, N\}$, which are given by ${ }^{3}$

$$
\pi_{i}^{\Delta}=\pi_{i} \Delta^{\frac{1}{2}} \quad \forall i \in\{0, \ldots, N\}
$$

The agent's action $p^{\Delta}$ in each period and the cost of his action will be evaluated as a function of the deviation of $p^{\Delta}$ from some standard $\hat{p}$, where $\hat{p} \in P, \hat{p} \gg 0$, is an action involving zero expected profits, i.e.

$$
\sum_{i=0}^{N} \hat{p}_{i} \pi_{i}^{\Delta}=\sum_{i=0}^{N} \hat{p}_{i} \pi_{i}=0
$$

\footnotetext{
${ }^{2}$ If the first-order conditions for the agent's maximization problem are sufficient as well as necessary, this is without loss of generality. Otherwise, having a payment $s_{i}$ strictly below the right hand side of (2) may serve to discourage the agent from some "far away" action $p^{\prime}$ involving $p_{i}^{\prime}>0$.

${ }^{3}$ In their discussion of the relation between multi-period discrete-time and continuous-time models, Holmström and Milgrom do not indicate that the possible outcome values in any one period must depend on the length of the period (1987, p. 318). Such a normalization is, however, implicit in their analysis as it underlies their subsequent appeal to the central limit theorem. For a more systematic discussion of the role played by the dependence of the potential values of period profits on period length, see the companion paper by Hellwig (1998a).
} 
for all $\Delta$. The effort cost in a period of length $\Delta$ if the agent chooses $p^{\Delta}$ is denoted by $c^{\Delta}\left(p^{\Delta}\right)$ which is defined by

$$
c^{\Delta}\left(p^{\Delta}\right) \equiv \Delta \cdot c\left(\hat{p}_{0}+\frac{p_{0}^{\Delta} \Leftrightarrow \hat{p}_{0}}{\Delta^{\frac{1}{2}}}, \ldots, \hat{p}_{N}+\frac{p_{N}^{\Delta} \Leftrightarrow \hat{p}_{N}}{\Delta^{\frac{1}{2}}}\right) .
$$

What is the point of this specification? There are $\frac{1}{\Delta}$ periods each of length $\Delta$. In order to keep total profits over the $\frac{1}{\Delta}$ periods comparable to expected profits in the static model we have to keep expected profits per period to an order of magnitude $\Delta$. Consider an action $p^{\Delta}$ that shifts probability mass from some low-profit outcome to some high-profit outcome as compared to the action $\hat{p}$. With profit levels proportional to $\Delta^{\frac{1}{2}}$ this raises expected profits by an order of magnitude $\Delta^{\frac{1}{2}}$. However, the agent's cost of this shift in probability mass is made to depend on $\Delta$ in such a way that the agent will keep the order of magnitude of such shifts ordinarily to $\Delta^{\frac{1}{2}}$. That is, if the isoquants of the cost function $c(\cdot)$ exhibit nonzero curvature, then the deviation of the action $p^{\Delta}$ that is actually chosen from the zero-expected-profits action $\hat{p}$ will be on the order of $\Delta^{\frac{1}{2}}$, and expected profits per period will be on the order of $\Delta$.

To make these ideas more precise, define

$$
\mu_{i}^{\Delta}=k_{i} \frac{p_{i}^{\Delta} \Leftrightarrow \hat{p}_{i}}{\Delta^{\frac{1}{2}}}
$$

for $i=1, \ldots, N$, with $k_{i}=\pi_{i} \Leftrightarrow \pi_{0}$. Note that if the agent chooses $p^{\Delta}$ in each period, then total expected profits in the period of length 1 are given by

$$
\frac{1}{\Delta} \sum_{i=0}^{N} p_{i}^{\Delta} \pi_{i}^{\Delta}=\frac{1}{\Delta} \sum_{i=0}^{N}\left(p_{i}^{\Delta} \Leftrightarrow \hat{p}_{i}\right)\left(\pi_{i} \Leftrightarrow \pi_{0}\right) \Delta^{\frac{1}{2}}=\sum_{i=0}^{N}\left(\pi_{i} \Leftrightarrow \pi_{0}\right) \frac{p_{i}^{\Delta} \Leftrightarrow \hat{p}_{i}}{\Delta^{\frac{1}{2}}}=\sum_{i=1}^{N} \mu_{i}^{\Delta} .
$$

For any $i$, the quantity $\mu_{i}^{\Delta}$ in (7) and (8) can be interpreted as the contribution to expected profits per unit of "real time" that stems from the agent shifting probability mass from state of the world zero to state of the world $i$, relative to the standard set be the zeroexpected-profits action $\hat{p}$.

It will be useful to think of the agent choosing the vector $\mu^{\Delta}=\left(\mu_{1}^{\Delta}, \ldots, \mu_{N}^{\Delta}\right)$ in each period, which then determines an associated action $p^{\Delta}\left(\mu^{\Delta}\right)$ where for $i=1, \ldots, N$,

$$
\begin{aligned}
& p_{i}^{\Delta}\left(\mu^{\Delta}\right)=\hat{p}_{i}+\mu_{i}^{\Delta} \frac{\Delta^{\frac{1}{2}}}{k_{i}} \forall i \in\{1, \ldots, N\}, \\
& p_{0}^{\Delta}\left(\mu^{\Delta}\right)=1 \Leftrightarrow \sum_{i=1}^{N} p_{i}^{\Delta}=\hat{p}_{0} \Leftrightarrow \sum_{i=1}^{N} \mu_{i}^{\Delta} \frac{\Delta^{\frac{1}{2}}}{k_{i}} .
\end{aligned}
$$


Since $p^{\Delta}$ is a probability vector we have to restrict the agent's choice of $\mu^{\Delta}$ by

$$
\Leftrightarrow \frac{k_{i} \hat{p}_{i}}{\Delta^{\frac{1}{2}}} \leq \mu_{i}^{\Delta} \leq \frac{k_{i}\left(1 \Leftrightarrow \hat{p}_{i}\right)}{\Delta^{\frac{1}{2}}}
$$

for all $i \in\{1, \ldots, N\}$ and

$$
\Leftrightarrow \frac{1 \Leftrightarrow \hat{p}_{0}}{\Delta^{\frac{1}{2}}} \leq \sum_{i=1}^{N} \frac{\mu_{i}^{\Delta}}{k_{i}} \leq \frac{\hat{p}_{0}}{\Delta^{\frac{1}{2}}}
$$

Note that the set of $\mu^{\Delta}$ satisfying (11) and (12) increases and goes to the entire set $I R^{N}$ as $\Delta \rightarrow 0$.

The agent's cost from the choice $\mu^{\Delta}$ in a time period with length $\Delta$ is given by:

$$
\Delta \hat{c}\left(\mu^{\Delta}\right)=c^{\Delta}\left(p^{\Delta}\left(\mu^{\Delta}\right)\right)
$$

where by $(6)$

$$
c^{\Delta}\left(p^{\Delta}\left(\mu^{\Delta}\right)\right)=\Delta c\left(\hat{p}+\frac{p^{\Delta}\left(\mu^{\Delta}\right) \Leftrightarrow \hat{p}}{\Delta^{\frac{1}{2}}}\right)=\Delta c\left(\hat{p}_{0} \Leftrightarrow \sum_{i=1}^{N} \frac{\mu_{i}^{\Delta}}{k_{i}}, \hat{p}_{1}+\frac{\mu_{1}^{\Delta}}{k_{1}}, \ldots, \hat{p}_{N}+\frac{\mu_{N}^{\Delta}}{k_{N}}\right) .
$$

Note that for any given vector $\mu$ that is independent of $\Delta$ the argument of $c(\cdot)$ in (14) is also independent of $\Delta$. The tradeoff between the vector $\mu^{\Delta}=\left(\mu_{1}^{\Delta}, \ldots, \mu_{N}^{\Delta}\right)$ of contributions to expected profits per unit of "real time" that stem from shifting probability mass to outcomes $1, \ldots, N$ and the associated effort cost per unit of "real time" $\hat{c}\left(\mu^{\Delta}\right)$ is thus independent of $\Delta$.

We are now ready to prove our first result, which shows that given our specification of profits, costs, actions and probabilities it is indeed possible to compare the discrete-time models with different period lengths $\Delta$.

Proposition 1 Consider the discrete problem with $\frac{1}{\Delta}$ subintervals where profit levels are given by (4) and where the agent's effort cost as a function of $\mu^{\Delta}$ is given by (14) each period. If for $\tau=1, \ldots, \frac{1}{\Delta}$ the agent chooses $\mu^{\Delta, \tau}=$ $\left(\mu_{1}^{\Delta, \tau}, \ldots, \mu_{N}^{\Delta, \tau}\right)$, then expected gross profits are equal to $\Delta \sum_{\tau=1}^{1 / \Delta} \sum_{i=1}^{N} \mu_{i}^{\Delta, \tau}$, and effort costs are equal to $\Delta \sum_{\tau=1}^{1 / \Delta} \hat{c}\left(\mu^{\Delta, \tau}\right)$, where $\hat{c}(\cdot)$ is given by (13). In particular, if $\mu^{\Delta, \tau}=\mu$ regardless of $\tau$ and of the period length $\Delta$, then expected gross profits and total effort costs of the agent are independent of $\Delta$. 
The proposition shows that if the agent chooses a constant $\mu$, then expected profits and effort costs of the agent are independent of $\Delta$. In particular, the trade-off between expected profits and effort costs is not affected by the length of the period. To be sure, the implementation problem as seen by the principal will also depend on the variance and the other higher moments of the distribution of profits, all of which depend on $\Delta$. However, as will be shown in the next section, when $\Delta$ is small and the agent's action is close to the standard $\hat{p}$, these higher moments are close to being independent of $\mu$, and the incentive payments that are required to implement $p^{\Delta}(\mu)$ admit a simple approximation.

\section{Approximation of the Brownian Model}

So far we assumed that the agent can change his action only at discrete points in time. Now we are interested in the case where the agent can change his action continuously at any point in time. In the following we will derive the continuous case as the limit of the discrete model when $\Delta$ goes to 0 .

The following definitions will be useful. Let $\tilde{A}_{i}^{\Delta, \tau} \in\{0,1\}$ be a random variable such that $\tilde{A}_{i}^{\Delta, \tau}=1 \Leftrightarrow \tilde{\pi}^{\Delta, \tau}=\pi_{i}^{\Delta}, i \in\{0, \ldots N\}, \Delta \in\left\{\frac{1}{m}\right\}_{m=1, \ldots}, \tau \in\left\{1, \ldots, \frac{1}{\Delta}\right\}$. Clearly, $\operatorname{Prob}\left(\tilde{A}_{i}^{\Delta, \tau}=1\right)=p_{i}^{\Delta, \tau}$, and $\tilde{A}_{i}^{\Delta, \tau}=1$ implies $\tilde{A}_{j}^{\Delta, \tau}=0$ for all $j \neq i$. Thus, each $\tilde{A}_{i}^{\Delta, \tau}$ has a Bernoulli distribution, but $\widetilde{A}_{i}^{\Delta, \tau}$ and $\widetilde{A}_{j}^{\Delta, \tau}$ are not stochastically independent.

Let $s_{i}^{\Delta}$ be the incentive payment for outcome $i$ corresponding to (2) when the period length is $\Delta$, the effort cost function is given by (6), and the certainty equivalent of the agent's utility is 0 . Using (13) and (14) one can write $s_{i}^{\Delta}$ in terms of the vector $\mu^{\Delta}$ rather than the action $p^{\Delta}\left(\mu^{\Delta}\right)$ that corresponds to it. This yields:

$$
s_{i}^{\Delta}=\Delta \hat{c}\left(\mu^{\Delta}\right) \Leftrightarrow \frac{1}{r} \ln \left(1 \Leftrightarrow r \hat{c}_{i} k_{i} \Delta^{\frac{1}{2}}+r \sum_{j=0}^{N} p_{j}^{\Delta} \hat{c}_{j} k_{j} \Delta^{\frac{1}{2}}\right),
$$

where $\hat{c}_{i}=\frac{c_{i}-c_{0}}{k_{i}}$ is the partial derivative of $\hat{c}$ with respect to $\mu_{i}^{\Delta}, \hat{c}_{0}=0$, and use has been made of the fact that by (6) $c_{i}^{\Delta}=\Delta^{\frac{1}{2}} c_{i}$ for all $i$. (Clearly the convexity and differentiability assumption on $c(\cdot)$ imply that $\hat{c}(\cdot)$ is strictly convex and continuously differentiable on the set of all vectors $\mu=\left(\mu_{1}, \ldots, \mu_{N}\right)$ for which $\hat{p}_{0}>\sum_{i=1}^{N} \mu_{i} / k_{i}$ and $\hat{p}_{i}>\Leftrightarrow \mu_{i} / k_{i}$ for $i=1, \ldots, N$.) 
Using a Taylor series expansion of the logarithmic term in (15), the requisite incentive payment $s_{i}^{\Delta}$ is now approximated by:

$$
s_{i}^{\Delta}=\Delta \hat{c}\left(\mu^{\Delta}\right)+\left[\hat{c}_{i} k_{i} \Leftrightarrow \sum_{j=0}^{N} p_{j}^{\Delta}\left(\mu^{\Delta}\right) \hat{c}_{j} k_{j}\right] \Delta^{\frac{1}{2}}+\frac{r}{2}\left[\hat{c}_{i} k_{i} \Leftrightarrow \sum_{j=0}^{N} p_{j}^{\Delta}\left(\mu^{\Delta}\right) \hat{c}_{j} k_{j}\right]^{2} \Delta+O\left(\Delta^{\frac{3}{2}}\right)
$$

Suppose that the principal wants to implement the time path of actions $\left\{\mu^{\Delta, \tau}\right\}_{\tau=1, \ldots, \frac{1}{\Delta}}$. Then, still assuming that the certainty equivalent of the agent's utility has to be 0 , the total renumeration that has to be offered is given by

$$
\begin{aligned}
\tilde{s}^{\Delta}=\Delta & \sum_{\tau=1}^{1 / \Delta} \hat{c}\left(\mu^{\Delta, \tau}\right)+\sum_{\tau=1}^{1 / \Delta} \sum_{i=0}^{N} \tilde{A}_{i}^{\Delta, \tau} \Delta^{\frac{1}{2}}\left[\hat{c}_{i} k_{i} \Leftrightarrow \sum_{j=0}^{N} p_{j}^{\Delta}\left(\mu^{\Delta}\right) \hat{c}_{j} k_{j}\right] \\
& +\frac{r}{2} \sum_{\tau=1}^{1 / \Delta} \sum_{i=0}^{N} \tilde{A}_{i}^{\Delta, \tau} \Delta\left[\hat{c}_{i} k_{i} \Leftrightarrow \sum_{j=0}^{N} p_{j}^{\Delta}\left(\mu^{\Delta}\right) \hat{c}_{j} k_{j}\right]^{2}+\sum_{\tau=1}^{1 / \Delta} O\left(\Delta^{\frac{3}{2}}\right) \\
=\Delta & \sum_{\tau=1}^{1 / \Delta} \hat{c}\left(\mu^{\Delta, \tau}\right)+\sum_{\tau=1}^{1 / \Delta} \sum_{i=0}^{N} \hat{c}_{i}\left(\mu^{\Delta, \tau}\right)\left(\tilde{A}_{i}^{\Delta, \tau} \Leftrightarrow p_{i}^{\Delta}\left(\mu^{\Delta, \tau}\right)\right) k_{i} \Delta^{\frac{1}{2}} \\
& +\frac{r}{2} \sum_{\tau=1}^{1 / \Delta} \sum_{i=0}^{N} \tilde{A}_{i}^{\Delta, \tau}\left[\hat{c}_{i}\left(\mu^{\Delta, \tau}\right) k_{i} \Leftrightarrow \sum_{j=0}^{N} p_{j}^{\Delta}\left(\mu^{\Delta, \tau}\right) \hat{c}_{j}\left(\mu^{\Delta, \tau}\right) k_{j}\right]^{2} \Delta+O\left(\Delta^{\frac{1}{2}}\right)
\end{aligned}
$$

where we made use of the fact that $\sum_{i=0}^{N} \tilde{A}_{i}^{\Delta, \tau}=1$ for all $\tau \in\{1, \ldots, 1 / \Delta\}$. If we substitute $p_{j}^{\Delta}\left(\mu^{\Delta, \tau}\right)$ in the squared term by (9) and (10), use $\hat{c}_{0}=0$, and rearrange by putting the appropriate terms into $O\left(\Delta^{\frac{1}{2}}\right)$ we get

$$
\begin{aligned}
\tilde{s}^{\Delta}=\Delta & \sum_{\tau=1}^{1 / \Delta} \hat{c}\left(\mu^{\Delta, \tau}\right)+\sum_{\tau=1}^{1 / \Delta} \sum_{i=0}^{N} \hat{c}_{i}\left(\mu^{\Delta, \tau}\right)\left(\tilde{A}_{i}^{\Delta, \tau} \Leftrightarrow p_{i}^{\Delta}\left(\mu^{\Delta, \tau}\right)\right) k_{i} \Delta^{\frac{1}{2}} \\
& +\frac{r}{2} \sum_{\tau=1}^{1 / \Delta} \sum_{i=0}^{N} \tilde{A}_{i}^{\Delta, \tau}\left[\hat{c}_{i}\left(\mu^{\Delta, \tau}\right) k_{i} \Leftrightarrow \sum_{j=0}^{N} \hat{p}_{j} \hat{c}_{j}\left(\mu^{\Delta, \tau}\right) k_{j}\right]^{2} \Delta+O\left(\Delta^{\frac{1}{2}}\right)
\end{aligned}
$$

Equation (18) admits a simple interpretation: ${ }^{4}$ The first term reflects the total effort cost of the agent from choosing $\left\{\mu^{\Delta, \tau}\right\}, \tau=1, \ldots, \frac{1}{\Delta}$. The second term gives the appropriate incentives to the agent to actually choose this time path of actions. If outcome $i$ is realized in period $\tau$, this raises the agent's overall incentive payment by an amount $\hat{c}_{i}\left(\mu^{\Delta, \tau}\right) k_{i} \Delta^{\frac{1}{2}}$, reflecting the marginal cost of shifting probability mass towards outcome $i$. The expected value of this payment, $p_{i}^{\Delta}\left(\mu^{\Delta, \tau}\right) c_{i}^{\Delta}\left(\mu^{\Delta, \tau}\right) k_{i} \Delta^{\frac{1}{2}}$, is subtracted again because in expectedvalue terms the principal's payments to the agent depend only on individual-rationality

\footnotetext{
${ }^{4}$ See also Schättler and Sung (1993, p. 337).
} 
considerations. Thus the second term in (18) is proportional to the difference between the actual realization of this profit level and the expected realization given that the agent chooses $p_{i}^{\Delta}\left(\mu^{\Delta, \tau}\right)$. It is useful to simplify this term by defining

$$
\tilde{X}_{i}^{\Delta, \tau}=k_{i} \Delta^{\frac{1}{2}}\left(\tilde{A}_{i}^{\Delta, \tau} \Leftrightarrow p_{i}^{\Delta}\left(\mu^{\Delta, \tau}\right)\right)
$$

The third term is the risk premium that has to be paid to the agent to compensate him for the randomness of the second term. The last term reflects the approximation we are using; it vanishes as $\Delta$ goes to zero.

Given the way the per-period deviations $\tilde{X}_{i}^{\Delta, \tau}$ of realized from expected profits depend on the length of the period, we find it more convenient to work with the cumulative deviations

$$
\tilde{X}_{i}^{\Delta}(\tau)=k_{i} \Delta^{\frac{1}{2}} \sum_{\tau^{\prime}=1}^{\tau}\left[\tilde{A}_{i}^{\Delta, \tau^{\prime}} \Leftrightarrow p_{i}^{\Delta}\left(\mu^{\Delta, \tau^{\prime}}\right)\right]
$$

from period one to period $\tau$. For $i=1, \ldots, N, \tilde{X}_{i}^{\Delta}(\tau)$ can be thought of as the cumulative deviation (up to $\tau$ ) of realized profits under outcome $i$ from the expected value of these profits under the given policy of the agent. $\tilde{X}_{i}^{\Delta}(\tau)$ will be called the "stochastic process of cumulative deviations from the mean".

To embed the discrete-time model in continuous time, we use a linear interpolation to represent the process $\tilde{X}_{i}^{\Delta}(\tau)$ by a continuous-time process $X_{i}^{\Delta}(t)$ such that for $t \in[0,1]$ :

$$
X_{i}^{\Delta}(t)=\left(1 \Leftrightarrow \frac{t}{\Delta}+\left[\frac{t}{\Delta}\right]\right) \tilde{X}_{i}^{\Delta}\left(\left[\frac{t}{\Delta}\right]\right)+\left(\frac{t}{\Delta} \Leftrightarrow\left[\frac{t}{\Delta}\right]\right) \tilde{X}_{i}^{\Delta}\left(\left[\frac{t}{\Delta}\right]+1\right)
$$

where $\left[\frac{t}{\Delta}\right]$ denotes the greatest integer less than or equal to $\frac{t}{\Delta}$. Note that $X_{i}^{\Delta}(t)$ is a random function taking values in $C=C[0,1]$, the space of continuous functions on $[0,1]$.

For any deterministic time path of actions ${ }^{5}\left\{\mu^{\Delta, \tau}\right\}_{\tau=1, \ldots, \frac{1}{\Delta}}$ in the discrete-time model we use a continuous-time representation $\mu^{\Delta}(\cdot)$ where

$$
\mu^{\Delta}(t)=\mu^{\Delta,[t / \Delta]}
$$

We can now state our first main result:

\footnotetext{
${ }^{5}$ We restrict attention to deterministic time paths of actions. Since Holmström and Milgrom have shown that the agent will be induced by the optimal contract to take a constant deterministic action there is no need to consider stochastic controls explicitly at this stage.
} 
Theorem 1 Consider a sequence of discrete models with period length $\Delta$, $\Delta=1, \frac{1}{2}, \frac{1}{3}, \ldots$ Suppose that, as $\Delta \rightarrow 0$, the time path of actions $\mu^{\Delta}(t)$ converges uniformly to some continuous function $\mu(t), t \in[0,1]$ such that $\hat{p}_{0}>\sum_{i} \mu_{i}(t) / k_{i}$ and $\hat{p}_{i}>\Leftrightarrow \mu_{i}(t) / k_{i}$ for all $t$ and $i=1, \ldots, N$. Then, as $\Delta \rightarrow 0$,

(a) the stochastic process of cumulative deviations from the mean $X^{\Delta}(t)=$ $\left(X_{1}^{\Delta}(t), \ldots, X_{N}^{\Delta}(t)\right)$ converges in distribution to a process $X(\cdot)$ which is a driftless $N$-dimensional Brownian motion with covariance matrix

$$
\Sigma=\left(\begin{array}{cccc}
k_{1}^{2} \hat{p}_{1}\left(1 \Leftrightarrow \hat{p}_{1}\right) & \Leftrightarrow k_{1} k_{2} \hat{p}_{1} \hat{p}_{2} & \cdots & \Leftrightarrow k_{1} k_{N} \hat{p}_{1} \hat{p}_{N} \\
\Leftrightarrow k_{2} k_{1} \hat{p}_{2} \hat{p}_{1} & k_{2}^{2} \hat{p}_{2}\left(1 \Leftrightarrow \hat{p}_{2}\right) & \cdots & \Leftrightarrow k_{2} k_{N} \hat{p}_{2} \hat{p}_{N} \\
\vdots & \vdots & \ddots & \vdots \\
\Leftrightarrow k_{N} k_{1} \hat{p}_{N} \hat{p}_{1} & \Leftrightarrow k_{N} k_{2} \hat{p}_{N} \hat{p}_{2} & \cdots & k_{N}^{2} \hat{p}_{N}\left(1 \Leftrightarrow \hat{p}_{N}\right)
\end{array}\right)
$$

and starting point $X(0)=0$;

(b) the total cost to the agent converges to $\int_{0}^{1} \hat{c}(\mu(t)) d t$;

(c) the incentive payments that serve to implement $\mu^{\Delta}(t)$ with certainty equivalent $w$ converge in distribution to

$$
\tilde{s}=w+\int_{0}^{1} \hat{c}(\mu(t)) d t+\int_{0}^{1} \hat{c}^{\prime}(\mu(t)) d X+\frac{r}{2} \int_{0}^{1} \hat{c}^{\prime}(\mu(t)) \Sigma\left[\hat{c}^{\prime}(\mu(t))\right]^{T} d t
$$

where $\hat{c}^{\prime}(\cdot)=\left(\hat{c}_{1}(\cdot), \ldots, \hat{c}_{N}(\cdot)\right)$.

Remarks:

1. Theorem 1 is closely related to Theorem 6 of Holmström and Milgrom (1987) and Corollary 4.1 of Schättler and Sung (1993), who show that in the continuous model a time path of actions $\mu(\cdot)$ is implemented by an incentive scheme satisfying

$$
\begin{aligned}
s=w+ & \int_{0}^{1} \hat{c}(\mu(t)) d t+\int_{0}^{1} \hat{c}^{\prime}(\mu(t)) d Z \Leftrightarrow \int_{0}^{1} \hat{c}^{\prime}(\mu(t)) \mu(t) d t \\
& +\frac{r}{2} \int_{0}^{1} \hat{c}^{\prime}(\mu(t)) \Sigma\left[\hat{c}^{\prime}(\mu(t))\right]^{T} d t
\end{aligned}
$$

where $Z$ is a process given by the stochastic differential equation

$$
d Z=\mu d t+d B
$$


with initial condition $Z(0)=0$; here $B$ is an $N$-dimensional Brownian motion with covariance matrix $\boldsymbol{\Sigma}$ that is equivalent to the process $X$ in Theorem $1 .{ }^{6}$

In contrast, our Theorem 1 deals with the discrete model and shows that the incentive schemes that serve to implement the exogenously given sequence of control paths $\mu^{\Delta}(t), \Delta=1, \frac{1}{2}, \ldots$, converge to a continuous function (24). It does not show that in the continuous model the limit control path $\mu(t)$ is indeed implemented by the limit incentive scheme (24). However, under the assumptions imposed by Schättler and Sung (1993, Corollary 4.1), which we will impose and discuss in more detail in Section 5 , the limit of the incentive schemes $s^{\Delta}$ does indeed implement the limit of the control path $\mu^{\Delta}(t)$. Theorem 1 thus shows that the implementation condition (25) on incentive schemes and policies that Holmström and Milgrom obtained in their Brownian model can be interpreted as the limit of the corresponding conditions on incentive schemes and policies in approximating discrete-time models.

2. Discrete-time approximations of the continuous-time process $Z(\cdot)$ in $(25)$ can be given by specifying

$$
\tilde{Z}_{i}^{\Delta, \tau}=k_{i} \Delta^{\frac{1}{2}}\left[\tilde{A}_{i}^{\Delta, \tau} \Leftrightarrow \hat{p}_{i}\right]
$$

as the contribution to total profits stemming from outcome $i$ in excess of some "standard" that is given by the probability $\hat{p}_{i}$, and

$$
\tilde{Z}_{i}^{\Delta}(\tau)=k_{i} \Delta^{\frac{1}{2}}\left[\sum_{\tau^{\prime}=1}^{\tau} \tilde{A}_{i}^{\Delta, \tau^{\prime}} \Leftrightarrow \hat{p}_{i} \tau\right]
$$

as the cumulative contribution. Using the linear interpolation as before to obtain a continuous-time representation

$$
Z_{i}^{\Delta}(t)=\left(1 \Leftrightarrow \frac{t}{\Delta}+\left[\frac{t}{\Delta}\right]\right) \tilde{Z}_{i}^{\Delta}\left(\left[\frac{t}{\Delta}\right]\right)+\left(\frac{t}{\Delta} \Leftrightarrow\left[\frac{t}{\Delta}\right]\right) \tilde{Z}_{i}^{\Delta}\left(\left[\frac{t}{\Delta}\right]+1\right)
$$

of the process (28), we find that, by a simple corollary to Theorem 1(a), the processes $Z_{i}^{\Delta}(t)$ converge in distribution to the process $Z(\cdot)$ as $\Delta$ goes to zero. ${ }^{7}$

\footnotetext{
${ }^{6} \mathrm{Holmström}$ and Milgrom show that $(25)$ is necessary to implement $\mu(\cdot)$. Schättler and Sung consider a more general model and give conditions under which under which (25) is sufficient.

${ }^{7}$ Holmström and Milgrom provide a sketch of the relation between discrete and continuous models in terms of the processes $Z^{\Delta}(\tau)$ and $Z(\cdot)$ (without indicating the dependence of period profit levels on $\Delta)$. We have instead focussed on the processes $X^{\Delta}(\tau)$ and $X(\cdot)$ because these processes are of central importance in the application of Donsker's Theorem.
} 
To better understand the role of the "standard" $\hat{p}_{i}$ in the specification (27) and (28) note that if $\Delta$ is small, and if the agent chooses a constant $\mu$ in some small time interval $\left[t, t^{\prime}\right]$, we have

$$
\mathrm{E}\left(Z_{i}^{\Delta}\left(t^{\prime}\right) \Leftrightarrow Z_{i}^{\Delta}(t)\right) \approx k_{i} \Delta^{\frac{1}{2}}\left(p_{i}^{\Delta}(\mu) \Leftrightarrow \hat{p}_{i}\right) \frac{t^{\prime} \Leftrightarrow t}{\Delta}=\mu_{i}\left(t \Leftrightarrow t^{\prime}\right) .
$$

Thus, if the agent chooses $\hat{\mu}^{\tau}=(0, \ldots, 0)$ for all $\tau \in\left[t, t^{\prime}\right]$, then $p_{i}^{\Delta}(\mu)=\hat{p}_{i}$ in each period and the agent allocates his probability mass according to the standard. If $\mu_{i}>0\left(\mu_{i}<0\right)$ he puts more (less) weight on achieving state of the world $i$ rather than state of the world 0 (as compared to the standard set by $\hat{p}$ ). This is reflected in the account process $Z_{i}^{\Delta}(t)$ which in expectation measures how much weight the agent puts on achieving state $i$. Furthermore, we know from the proof of Proposition 1 that if the agent chooses a constant $\mu$, expected profits in time interval $\left[t, t^{\prime}\right]$ are given by

$$
\sum_{\tau=[t / \Delta]}^{\left[t^{\prime} / \Delta\right]} \sum_{i=0}^{N} p_{i}^{\Delta} \pi_{i}^{\Delta}=\left(\left[t^{\prime} / \Delta\right] \Leftrightarrow[t / \Delta]\right) \cdot \sum_{i=1}^{N} \mu_{i} \approx\left(t^{\prime} \Leftrightarrow t\right) \sum_{i=1}^{N} \mu_{i} .
$$

Recall that $\sum_{i=0}^{N} \hat{p}_{i} \pi_{i}=0$. Hence, $\mu_{i}$ is simply the expected contribution to total profits that stems from the realizations of state of the world $i$ in excess of the standard set by $\hat{p}$, and the account $Z_{i}^{\Delta}(t)$ measures the actual contribution over time.

3. In the continuous-time limit the agent controls the drift rate but not the higher moments of the cumulative output process. Note that he does control the entire distribution of the cumulative-output process in the discrete-time models. However, when $\Delta$ is small, implementation of a given drift rate process involves actions $p^{\Delta}(\mu(\cdot))$ close to the constant action $\hat{p}$, the difference in any period being of order $\Delta^{\frac{1}{2}}$. Because of this convergence of the path of actions to the constant path with value $\hat{p}$, the second moments of the cumulative-returns process in the limit are fully determined by $\hat{p}$, and the higher moments vanish. The critical action vector $\hat{p}$ thus serves a dual function in our model: First, as an action vector with a zero profit expectation it provides a base deviations from which measure the impact of behaviour on expected profits (see equation (30)). Second, it determines the "noise" of the agency problem in continuous time. 
Theorem 1 takes the discrete model as given and shows how to obtain a Brownian model as the limit of a sequence of these discrete models. We could have proceeded the other way round, asking whether it is possible to approximate a given Brownian model with some sequence of discrete models. The following result, which is an immediate corollary to Theorem 1, gives an answer to this question.

Corollary 1 Let $B$ be a given $N$-dimensional Brownian motion with zero drift and covariance matrix $\boldsymbol{\Sigma}$. Suppose that if the agent chooses a control process $\mu(\cdot)$, then the agent's cumulative costs are given by the differential equation

$$
d c=\hat{c}(\mu(t)) d t
$$

and the disturbance process is $B$. If there exist real numbers $k_{1}, \ldots, k_{N}$ and positive real numbers $\hat{p}_{1}, \ldots, \hat{p}_{N}$ with $\sum_{i=1}^{N} \hat{p}_{i} \leq 1$ such that $\boldsymbol{\Sigma}$ can be written as in (23), then to this continuous time model there corresponds a discrete-time model with period length $\Delta$ in which

- there are $N+1$ possible profit levels in each period which are given by

$$
\begin{aligned}
& \pi_{0}^{\Delta}=\Leftrightarrow \Delta^{\frac{1}{2}} \sum_{i=1}^{N} \hat{p}_{i} k_{i} \\
& \pi_{i}^{\Delta}=\Delta^{\frac{1}{2}}\left(k_{i} \Leftrightarrow \sum_{i=1}^{N} \hat{p}_{i} k_{i}\right)
\end{aligned}
$$

- the probability $p_{i}^{\Delta}\left(\mu^{\Delta}\right)$ of profit level $i$ is given by (9) and (10), with $\mu^{\Delta,[t / \Delta]}=\mu([t / \Delta])$,

- and the cost to the agent in each period is given by $c^{\Delta}\left(p^{\Delta}\left(\mu^{\Delta}\right)\right)=\Delta \hat{c}\left(\mu^{\Delta}\right)$, such that the continuous model is the limit of this discrete model (in the sense of Theorem 1) as $\Delta$ approaches 0 .

Corollary 2 requires that the covariance matrix $\boldsymbol{\Sigma}$ of the $N$-dimensional Brownian motion can be written as in (23). If $N=1$, this is always possible. If $N>1$, this condition implies a restriction on the set of Brownian models that can be approximated by a sequence of discrete models. This restriction stems from the fact that in the discrete model only one state of the world can materialize in each period. This implies that the 
accounts $\tilde{A}_{i}^{\Delta, \tau}$ and thus also the accounts $\tilde{X}_{i}^{\Delta, \tau}$ have a special covariance structure. For example, it is impossible that the $\tilde{X}_{i}^{\Delta, \tau}$ are stochastically independent. Therefore this restriction is very natural.

In the entire analysis here, the dimension $N$ of the Brownian motion has referred to the number of different profit levels that can be distinguished. Holmström and Milgrom (1987, p. 322) offer a second interpretation according to which $N$ refers to different activities of the agent. For example, if $N=2$, account $X_{1}$ could be a measure of revenues, while $X_{2}$ could be a measure of costs. Thus, $\mu_{1}$ reflects the agent's effort to increase revenues, while $\mu_{2}$ reflects his effort to reduce costs. With this interpretation the Brownian model could have any variance-covariance structure. It is possible to show that any $N$ dimensional Brownian motion model (with an arbitrary covariance matrix $\boldsymbol{\Sigma}$ ) can be approximated by a discrete model, if we extend the dimension of the discrete model to $2^{N} \Leftrightarrow 1$. However, we do not want to go into the details of this approximation here. ${ }^{8}$

\section{The Convergence of Optimal Control Paths and Incentive Schemes}

Theorem 1 is not quite satisfactory in that it takes the sequence of control paths $\mu^{\Delta}(\cdot)$ and their convergence behaviour as given. However $\mu^{\Delta}(\cdot)$ is chosen endogenously by the agent in response to the incentive scheme $s^{\Delta}$. Moreover $s^{\Delta}$ and $\mu^{\Delta}(\cdot)$ together are chosen endogenously, subject to incentive compatibility, by the principal and agent when they initially agree on a contract. One may therefore wonder how relevant Theorem 1 still is once the endogenity of $s^{\Delta}$ and $\mu^{\Delta}(\cdot)$ is taken into account.

\footnotetext{
${ }^{8}$ To sketch the basic idea suppose that the Brownian model is two-dimensional and that $\mu_{1}$ is the drift rate of revenues while $\mu_{2}$ is the drift rate of costs. To approximate this model by a sequence of discrete models we need at least two different levels of revenues $R \in\left\{R_{1}, R_{2}\right\}$ and two levels of costs $C \in\left\{C_{1}, C_{2}\right\}$. Thus, in each period there are four different possible outcomes $\left\{\left(R_{1}, C_{1}\right),\left(R_{1}, C_{2}\right),\left(R_{2}, C_{1}\right),\left(R_{2}, C_{2}\right)\right\}$, which means that there are $2^{2}-1=3$ different accounts. With this three-dimensional model we can approximate any variance-covariance matrix in the two-dimensional continuous model by choosing the correlation between the Bernoulli distributions over $\left\{R_{1}, R_{2}\right\}$ and $\left\{C_{1}, C_{2}\right\}$ appropriately. If we want to allow for, say, $M_{r}$ levels of revenues and $M_{c}$ levels of costs, we need a model with $M_{r} \cdot M_{c}$ possible outcomes and hence $M_{r} \cdot M_{c}-1$ different accounts. The problem of getting from these $M_{r} \cdot M_{c}-1$ different accounts to incentive schemes that are defined in terms of just revenue and cost aggregates is then the same as the problem of aggregation accross accounts that is studied in Sections 5 and 6 .
} 
Two issues arise. First, for some incentive schemes it will be optimal for the agent to choose a control path that is not simply a continuous function of $t$, but that may also depend on the realizations of the profit process up to time $t$. Second, even if $\mu^{\Delta}(\cdot)$ is a history-independent constant, it is not clear that the sequence $\mu^{\Delta}(\cdot)$ should have a convergent subsequence.

Of these two issues, the first one is unproblematic. History dependence of the control strategies causes technical difficulties because the variables $\tilde{X}_{i}^{\Delta, \tau}$ in (19) are no longer independent, but in spite of these difficulties Theorem 1 can be extended to control paths $\mu^{\Delta}(\cdot)$ that are predictable functions of $t$ and the history up to $t$, for details see Appendix B. More importantly, Holmström and Milgrom (1987, Theorem 5) have shown that in the repeated discrete model there always is an optimal solution to the principal's problem in which he induces the agent to take the same action in each period, regardless of prior history. If $\mu^{\Delta *}$ is such a constant optimal control path in the discrete model with period length $\Delta$, and if the sequence $\left\{\mu^{\Delta *}\right\}$ has a convergent subsequence, Theorem 1 can automatically be applied to this subsequence.

However without additional assumptions, a sequence $\left\{\mu^{\Delta *}\right\}$ of constant optimal control paths in the discrete models will not necessarily have a convergent subsequence. Indeed in the following section we shall come across a fairly natural example in which $\mu^{\Delta *}$ goes out of bounds, with some of its components $\mu_{i}^{\Delta *}$ going to $+\infty$ and others going to $\Leftrightarrow \infty$ as $\Delta$ goes to zero. Such a possibility can be ruled out by additional assumptions, but then one must worry about the compatibility of such assumptions with the specification underlying Theorem 1.

To see the issue, suppose for example that the vector $\mu^{\Delta}$ must always be chosen from a product $K=\prod_{i=1}^{N}\left[\underline{m}_{i}, M_{i}\right]$ of compact intervals. With this additional assumption a sequence $\left\{\mu^{\Delta *}\right\}$ of constant optimal control paths in the discrete models will obviously have a subsequence that converges to a limit $\mu^{*}$. Moreover the first two statements of Theorem 1 will be satisfied for this subsequence, i.e. the process $X^{\Delta}(\cdot)$ of cumulative deviations from the mean converges in distributions to the driftless Brownian motion $X(\cdot)$ with covariance matrix $\Sigma$ and the agent's total cost converges to $\hat{c}\left(\mu^{*}\right)$. However the requirement that $\mu^{\Delta} \in K$ will modify the agent's incentive compatibility conditions and hence the relation between desired actions and required incentive payments; this throws 
doubt on statement (c) of Theorem 1.

In choosing his action $p \in P$, the agent now faces the additional constraints $\underline{p}_{i}^{\Delta} \leq$ $p_{i} \leq P_{i}^{\Delta}$, where $\underline{p}_{i}^{\Delta}=\hat{p}_{i}+\Delta^{\frac{1}{2}} \cdot \min \left\{\underline{m}_{i} / k_{i}, M_{i} / k_{i}\right\}$ and $P_{i}^{\Delta}=\hat{p}_{i}+\Delta^{\frac{1}{2}} \cdot \max \left\{\underline{m}_{i} / k_{i}, M_{i} / k_{i}\right\}$, $i=1, \ldots, N$. Taking account of these constraints in the agent's first-order conditions, one finds that the incentive-compatibility condition (19) must be rewritten as

$$
s_{i}^{\Delta}=\Delta \hat{c}\left(\mu^{\Delta}\right) \Leftrightarrow \frac{1}{r} \ln \left[1 \Leftrightarrow\left(r \hat{c}_{i} k_{i} \Delta^{\frac{1}{2}} \Leftrightarrow u_{i}^{\Delta}+v_{i}^{\Delta}\right)+\sum_{i=1}^{N}\left(r \hat{c}_{i} k_{i} \Delta^{\frac{1}{2}} \Leftrightarrow u_{i}^{\Delta}+v_{i}^{\Delta}\right)\right]
$$

where $u_{i}^{\Delta} \geq 0$ and $v_{i}^{\Delta} \geq 0$ are the Kuhn-Tucker multipliers of the constraints $\underline{p}_{i}^{\Delta} \leq p_{i}$ and $p_{i} \leq P_{i}^{\Delta}$ in the agent's problem, with complementary slackness requiring $u_{i}^{\Delta}\left(p_{i} \Leftrightarrow\right.$ $\left.\max \left\{0, \underline{p}_{i}^{\Delta}\right\}\right)=0$ and $v_{i}^{\Delta}\left(P_{i}^{\Delta} \Leftrightarrow p_{i}\right)=0$ for $i=1, \ldots, N$. Given the appearance of the Kuhn-Tucker multipliers $u_{i}^{\Delta}$ and $v_{i}^{\Delta}$ in (35), it is not clear that the approximation procedure of the preceding section can be used; for instance, a Taylor approximation of the logarithmic term in (35) would make sense only if $u_{i}^{\Delta}$ and $v_{i}^{\Delta}$ were known to go to zero as the continuous-time model is approached.

Holmström and Milgrom try to avoid this difficulty by assuming that optimal actions always lie in the interior of the admissible set. Presumably such an assumption is justified if the effort cost function satisfies suitable Inada conditions. Unfortunately such Inada conditions are not compatible with the uniform boundedness of the derivatives of the effort cost function $\hat{c}(\cdot)$ which has been used extensively in the proof of Theorem $1(\mathrm{c})$. Therefore we prefer to tackle the problem posed by the Kuhn-Tucker multiplers in (35) directly, without trying to rule boundary actions out. As it turns out, this can be done without any substantive change in the model.

From the principal's perspective, the appearance of the Kuhn-Tucker multipliers $u_{i}^{\Delta}$, $v_{i}^{\Delta}$ in (35) reflects the fact that when the action $p=p^{\Delta}\left(\mu^{\Delta}\right)$ is at the boundary of the admissible action set, there is more than one incentive scheme that will implement this action, so he must consider which of these incentive schemes is cheapest for him. For example suppose that $N=1, \pi_{1}>\pi_{0}$, and consider the action $p$ which assigns the smallest possible mass to the outcome 1, i.e., let $p=p^{\Delta}\left(\underline{m}_{1}\right)$. This action is implemented by any incentive scheme $\left(s_{0}^{\Delta}, s_{1}^{\Delta}\right)$ that satisfies $s_{1}^{\Delta} \Leftrightarrow s_{0}^{\Delta} \leq \frac{1}{r}\left[\ln \left(1+r p_{1} \hat{c}_{1} k_{1} \Delta^{\frac{1}{2}}\right) \Leftrightarrow \ln \left(1 \Leftrightarrow r p_{0} \hat{c}_{1} k_{1} \Delta^{\frac{1}{2}}\right)\right]$ and that is individually rational for the agent, the point being that the principal does not have to provide incentives for outcome 1 because the agent is unable to lower $p_{1}$ below the 
stipulated level anyway. Among the different schemes that implement the action $p^{\Delta}\left(\underline{m}_{1}\right)$, the scheme $s_{1}^{\Delta}=s_{0}^{\Delta}=\Delta \hat{c}\left(\underline{m}_{1}\right)$ is cheapest for the principal; it just compensates the agent for his cost and involves no risk premium.

Different incentive schemes that implement the same action will involve different values of the Kuhn-Tucker multipliers in (35). Using the two-outcome example one easily verifies that not all these Kuhn-Tucker multipliers will be small if $\Delta$ is small, so for some of the incentive schemes implementing the action $p^{\Delta}\left(\underline{m}_{1}\right)$, no analogue of Theorem $1(\mathrm{c})$ can be given. However in the two-outcome example one also sees that the incentive scheme which is cheapest for the principal, namely $s_{1}^{\Delta}=s_{0}^{\Delta}=\Delta \hat{c}\left(\underline{m}_{1}\right)$, involves Kuhn-Tucker multipliers $u_{1}^{\Delta}=r \hat{c}_{1} k_{1} \Delta^{\frac{1}{2}}$ and $v_{1}^{\Delta}=0$, which do become small as $\Delta$ goes to zero.

This last observation reflects a general principle. Quite generally the principal's concern for minimizing implementation costs leads to the use of incentive schemes involving payments with a mean on the order of $\Delta$ and deviations from the mean on the order of $\Delta^{\frac{1}{2}}$, so as $\Delta$ goes to zero an argument analogous to the one underlying Theorem 1 can be given. This provides the key to the convergence behavior of optimal control paths and incentive schemes, which we now discuss.

Consider the principal's optimization problem in the discrete model with period length $\Delta$. By Theorem 5 of Holmström and Milgrom, we can restrict attention to constant controls. A constant control $\mu^{\Delta} \in K$ in each period, with associated incentive scheme $\tilde{s}^{\Delta}$, will be optimal for the principal if it maximizes his expected payoff

$$
U^{P \Delta}=\sum_{i=1}^{N} \mu_{i}^{\Delta} \Leftrightarrow E\left(\tilde{s}^{\Delta}\right)
$$

under the given incentive compatibility and participation constraints. In view of the agent's first order condition (35), incentive schemes may be assumed to satisfy

$$
\tilde{s}^{\Delta}=\sum_{\tau=1}^{1 / \Delta} \sum_{i=0}^{N} \tilde{A}_{i}^{\Delta, \tau}\left(w+s_{i}^{\Delta}\right)
$$

where $w$ is the agent's certainty equivalent and, for each $i, s_{i}^{\Delta}\left(\mu^{\Delta}\right)$ satisfies (35) for a suitable set of Kuhn-Tucker multipliers $u_{i}^{\Delta}, v_{i}^{\Delta}$. It is convenient to write the Kuhn-Tucker multipliers in (35) in the form $r \Delta^{\frac{1}{2}} k_{i} \hat{u}_{i}^{\Delta}, r \Delta^{\frac{1}{2}} k_{i} \hat{v}_{i}^{\Delta}$, where for $i=1, \ldots, N, \hat{u}_{i}^{\Delta} \geq 0, \hat{v}_{i}^{\Delta} \geq 0$, and

$$
\hat{u}_{i}^{\Delta}\left(\mu_{i}^{\Delta} \Leftrightarrow \underline{m}_{i}\right)=\hat{v}_{i}^{\Delta}\left(M_{i} \Leftrightarrow \mu_{i}^{\Delta}\right)=0
$$


With this change of notation (35) can be rewritten as:

$$
\begin{aligned}
s_{i}^{\Delta}\left(\mu^{\Delta}\right) & =\Delta \hat{c}\left(\mu^{\Delta}\right) \\
& \Leftrightarrow \frac{1}{r} \ln \left[1 \Leftrightarrow r\left(\hat{c}_{i} \Leftrightarrow \hat{u}_{i}^{\Delta}+\hat{v}_{i}^{\Delta}\right) k_{i} \Delta^{\frac{1}{2}}+r \sum_{j=1}^{N} p_{j}^{\Delta}\left(\mu^{\Delta}\right)\left(\hat{c}_{j} \Leftrightarrow \hat{u}_{j}^{\Delta}+\hat{v}_{j}^{\Delta}\right) k_{j} \Delta^{\frac{1}{2}}\right]
\end{aligned}
$$

Upon substituting from (37) and (39) and using the fact that $E \tilde{A}_{i}^{\Delta, \tau}=p_{i}^{\Delta}\left(\mu^{\Delta}\right)$ we can write the principal's objective as:

$$
\begin{aligned}
& U^{P \Delta}\left(\mu^{\Delta}, \hat{u}_{1}^{\Delta}, \hat{v}_{1}^{\Delta}, \ldots, \hat{u}_{N}^{\Delta}, \hat{v}_{N}^{\Delta}\right)=\sum_{i=1}^{N} \mu_{i}^{\Delta} \Leftrightarrow w \Leftrightarrow \hat{c}\left(\mu^{\Delta}\right) \\
& \quad+\frac{1}{r \Delta} \sum_{i=1}^{N} p_{i}^{\Delta}\left(\mu^{\Delta}\right) \ln \left[1 \Leftrightarrow r\left(\hat{c}_{i} \Leftrightarrow \hat{u}_{i}^{\Delta}+\hat{v}_{i}^{\Delta}\right) k_{i} \Delta^{\frac{1}{2}}+r \sum_{j=1}^{N} p_{j}^{\Delta}\left(\mu^{\Delta}\right)\left(\hat{c}_{j} \Leftrightarrow \hat{u}_{j}^{\Delta}+\hat{v}_{j}^{\Delta}\right) k_{j} \Delta^{\frac{1}{2}}\right]
\end{aligned}
$$

In the discrete-time model with period length $\Delta$, the principal's problem reduces to maximizing (40) with respect to $\mu^{\Delta} \in K$ and $u_{i}^{\Delta} \in V, v_{i}^{\Delta} \in V, i=1, \ldots, N$, subject to the complementary slackness condition (38) and the incentive compatibility condition:

$$
\Leftrightarrow \sum_{i=1}^{N} p_{i}^{\Delta}\left(\mu^{\Delta}\right) e^{-r\left(s_{i}^{\Delta}-\Delta \hat{c}\left(\mu^{\Delta}\right)\right)} \geq \Leftrightarrow \sum_{i=1}^{N} p_{i}^{\Delta}\left(\mu^{\prime}\right) e^{-r\left(s_{i}^{\Delta}-\Delta \hat{c}\left(\mu^{\prime}\right)\right)}
$$

which for incentive schemes satisfying (39) is equivalent to

$$
\begin{aligned}
\Leftrightarrow 1 \geq & \Leftrightarrow \sum_{i=1}^{N} p_{i}^{\Delta}\left(\mu^{\prime}\right) e^{-r \Delta\left(\hat{c}\left(\mu^{\Delta}\right)-\hat{c}\left(\mu^{\prime}\right)\right)} \\
& \cdot\left(1 \Leftrightarrow r\left(\hat{c}_{i} \Leftrightarrow \hat{u}_{i}^{\Delta}+\hat{v}_{i}^{\Delta}\right) k_{i} \Delta^{\frac{1}{2}}+r \sum_{j=1}^{N} p_{j}^{\Delta}\left(\mu^{\Delta}\right)\left(\hat{c}_{j} \Leftrightarrow \hat{u}_{j}^{\Delta}+\hat{v}_{j}^{\Delta}\right) k_{j} \Delta^{\frac{1}{2}}\right)
\end{aligned}
$$

for all $\mu^{\prime} \in K$.

If the first-order condition (39) was sufficient as well as necessary for the implementation of $\mu^{\Delta}$ through the incentive scheme $\tilde{s}^{\Delta}$, the incentive constraint (42) would be redundant. However because of the convexity of the exponential function, (41) is typically stronger than the agent's first-order condition (39) so (42) is not redundant. Even so it is instructive to consider what happens when the principal replaces the global incentive compatibility constraint (41) by the first-order condition (39), i.e., when he maximizes (40) subject to the complementary slackness conditions (38) without paying attention to (41). For this "relaxed problem of the principal" we obtain: 
Proposition 2 Let $\mu^{\Delta} \in K$ be given and suppose that $\mu_{i}^{\Delta} \geq 0, \hat{v}_{i}^{\Delta} \geq 0$, $i=1, \ldots, N$, maximize (40) subject to the complementary slackness condition (38). Then $\hat{u}_{i}^{\Delta} \leq \gamma\left(\mu^{\Delta}\right) / k_{i}$ and $\hat{v}_{i}^{\Delta} \leq \gamma\left(\mu^{\Delta}\right) / k_{i}, i=1, \ldots, N$, where $\gamma\left(\mu^{\Delta}\right):=$ $\max _{i j}\left(k_{i} \hat{c}_{i}\left(\mu^{\Delta}\right) \Leftrightarrow k_{j} \hat{c}_{j}\left(\mu^{\Delta}\right)\right)$.

Proposition 2 suggests that the principal will optimally choose the incentive scheme $\tilde{s}^{\Delta}$ so that the Kuhn-Tucker multipliers for the agent's maximization problem will be commensurate to marginal costs and will have the order of magnitude $\Delta^{\frac{1}{2}}$. Unfortunately we cannot immediately use this result because the global incentive constraint (41) is not generally implied by the agent's first-order condition, and (42) is not in general redundant. However, as $\Delta$ goes to zero, this concern becomes less and less important, the difference between the incentive compatibility condition (41) and the first-order condition (38) disappears, and the incentive constraint (42) ceases to impose any additional constraints. Formally we have:

Proposition 3 For $\Delta=1, \frac{1}{2}, \ldots$, let $I C(\Delta)$ be the set of vectors $\left(\mu^{\Delta}, \underline{\hat{u}}^{\Delta}, \underline{\hat{v}}^{\Delta} \in\right.$ $K \times I R_{+}^{N} \times R_{+}^{N}$ that satisfy the incentive constraint (42) as well as the complementary slackness conditions (40). As $\Delta$ converges to zero, $I C(\Delta)$ converges (in the Hausdorff topology) to the set of vectors $(\mu, \underline{\hat{u}}, \underline{\hat{v}}) \in K \times I_{+}^{N} \times R_{+}^{N}$ that satisfy

$$
\hat{u}_{i}\left(\mu_{i} \Leftrightarrow \underline{m}_{i}\right)=\hat{v}_{i}\left(M_{i} \Leftrightarrow \mu_{i}\right)=0
$$

for $i=1, \ldots, N$.

Given Propositions 2 and 3, another application of the maximum theorem yields:

Theorem 2 Let $\left\{\mu^{\Delta *}\right\}$ be a sequence of constant optimal control paths that the principal wants to implement in the discrete model with period length $\Delta=$ $1, \frac{1}{2}$... when $\mu^{\Delta}$ is constrained to the set $K=\prod_{i=1}^{N}\left[\underline{m}_{i}, M_{i}\right]$. Let $\hat{u}_{i}^{\Delta *}, \hat{v}_{i}^{\Delta *}, i=$ $1, \ldots, N$, be the normalized Kuhn-Tucker multipliers induced by the corresponding optimal incentive schemes. The sequence $\left\{\mu^{\Delta *}, \hat{u}_{1}^{\Delta *}, \hat{v}_{1}^{\Delta *}, \ldots, \hat{u}_{N}^{\Delta *}, \hat{v}_{N}^{\Delta *}\right\}$ has a subsequence which converges to a limit $\left(\mu^{*}, \hat{u}_{1}^{*}, \hat{v}_{1}^{*}, \ldots, \hat{u}_{N}^{*}, \hat{v}_{N}^{*}\right)$. Moreover, $\mu^{*}$ is a constant optimal control path and the incentive scheme $s^{*}$ is given 
by

$$
s^{*}(X)=w+\hat{c}\left(\mu^{*}\right)+\sum_{i=1}^{N}\left(\hat{c}_{i}\left(\mu^{*}\right) \Leftrightarrow \hat{u}_{i}^{*}+\hat{v}_{i}^{*}\right) X_{i}(1)+\frac{r}{2} \sum_{i=1}^{N} \sum_{j=1}^{N}\left(\hat{c}_{i} \Leftrightarrow \hat{u}_{i}^{*}+\hat{v}_{i}^{*}\right) \sigma_{i j}\left(\hat{c}_{i} \Leftrightarrow \hat{u}_{i}^{*}+v_{i}^{*}\right),
$$

with $\left(\sigma_{i j}\right)=\Sigma$ given by (23), which is an optimal incentive scheme in the continuous model.

Turning to the optimal incentive schemes, we note that (40) can be rewritten in the linear form:

$$
\tilde{s}^{\Delta}=\alpha_{0}^{\Delta}+\sum_{i=1}^{N} \alpha_{i}^{\Delta} X_{i}^{\Delta}(1)
$$

where $\alpha_{0}^{\Delta}=w+s_{0}^{\Delta}\left(\mu^{\Delta}\right)$, and, for $i=1, \ldots, N, \alpha_{i}^{\Delta}=\left[s_{i}^{\Delta}\left(\mu^{\Delta}\right) \Leftrightarrow s_{0}^{\Delta}\left(\mu^{\Delta}\right)\right] / k_{i} \Delta^{\frac{1}{2}}$ and $X_{i}^{\Delta}$ is the process of cumulative deviations from the mean that was defined in (20) and (21). From Theorem 2 one immediately obtains:

Corollary 2 For $\Delta=1, \frac{1}{2}, \ldots$, let $\left(\alpha_{0}^{\Delta}, \alpha_{1}^{\Delta}, \ldots, \alpha_{N}^{\Delta}\right)$ be the parameters of an optimal incentive scheme implementing a constant optimal control $\mu^{\Delta *}$ when controls are restricted to the compact set $K=\prod_{i=1}^{N}\left[\underline{m}_{i}, M_{i}\right]$. The sequence $\left\{\alpha_{0}^{\Delta}, \alpha_{1}^{\Delta}, \ldots, \alpha_{n}^{\Delta}\right\}$ has a subsequence which converges to a limit $\left(\alpha_{0}, \alpha_{1}, \ldots, \alpha_{N}\right)$. The limit $\left(\alpha_{0}, \alpha_{1}, \ldots, \alpha_{N}\right)$ defines an optimal incentive scheme

$$
\tilde{s}=\alpha_{0}+\sum_{i=1}^{N} \alpha_{i} X_{i}(1)
$$

in the continuous model. The coefficients $\alpha_{i}, i=1, \ldots, N$, satisfy $\alpha_{i}=$ $\hat{c}_{i}\left(\mu^{*}\right) \Leftrightarrow u_{i}^{*}+v_{i}^{*}$ where $\mu^{*}$ and $u_{i}^{*}, v_{i}^{*}, i=1, \ldots, N$, are the corresponding limits of $\mu^{\Delta *}$ and $u_{i}^{\Delta *}, v_{i}^{\Delta *}$ along the convergent subsequence. ${ }^{9}$

\footnotetext{
${ }^{9}$ In Corollary 1, as in Theorem 1, one can replace the processes $X_{i}^{\Delta}(\cdot)$ and $X_{i}(\cdot)$ by the processes $Z_{i}^{\Delta}(\cdot)$ and $Z_{i}(\cdot)$ that were used by Holmström and Milgrom and that are defined in (26) - (29). In terms of these processes, (45) and (46) become $\tilde{s}^{\Delta}=\hat{\alpha}_{0}^{\Delta}+\sum_{i=1}^{N} \hat{\alpha}_{i}^{\Delta} Z_{i}^{\Delta}(1)$, and $\tilde{s}=\hat{\alpha}_{0}+\sum_{i=1}^{N} \alpha_{i} Z_{i}(1)$, where, for $i=1, \ldots, N, \alpha_{i}^{\Delta}$ and $\alpha_{i}$ are the same as before and $\hat{\alpha}_{0}^{\Delta}=\alpha_{0}^{\Delta}-\sum_{i=1}^{N} \mu_{i}^{\Delta *}, \hat{\alpha}_{0}=\alpha_{0}-\sum_{i=1}^{N} \mu_{i}^{*}$.
} 


\section{Linearity in Aggregates}

So far the optimal incentive schemes in the discrete multi-period and in the corresponding Brownian model are linear only in accounts, not in total profits. Specifically, total incentive payments in (45) are equal to a constant plus $\sum_{i=1}^{N} \hat{c}_{i}\left(\mu^{*}\right) X_{i}(1)$; unless $\hat{c}_{i}\left(\mu^{*}\right)$ is the same for all $i=1, \ldots, N$, this is not representable as a function of $\sum_{i=1}^{N} X_{i}(1)$, the total contribution to profits from having outcomes other than 0 . In particular, if $N$ is large, i.e., if there are many different possible profit levels in each period, the corresponding Brownian motion is of high dimension and the optimal incentive scheme may be very complex, much more complex than real world contracts that are often linear in aggregates, such as total profits.

As noted by Holmström and Milgrom, the preceding remarks are moot if $N=1$, and the static model involves just two possible outcomes. In this case, the aggregate bonus for outcomes other than 0 reduces to $\hat{c}_{1} X_{1}(1)$ and the problem of aggregation across outcomes other than 0 does not arise. Since any Brownian motion can be represented as a limit of binomial processes, it is sometimes believed that this observation is enough to support the linearity of incentive schemes in Brownian models. For a unidimensional Brownian motion, this is of course correct. However, Theorem 1 and Corollary 2 show that the unidimensional Brownian motion does not actually yield the appropriate limit for multi-period problems with multinomial rather than binomial processes. The underlying structure of the intertemporal agency problem - in continuous as well as discrete time - is given by the function $\hat{c}(\cdot)$ which indicates the tradeoff between the vector of contributions of the different accounts to profits per unit of "real time" and the agent's effort costs per unit of "real time". The dimension $N$ of the domain of this function is an essential feature of the economic situation. This dimension corresponds to the cardinality of the outcome set in the static model as well as the dimension of the Brownian motion in the continuous-

time model. For $N>1$, the observation that unidimensional Brownian motions can be represented as limits of binomial processes is therefore irrelevant.

As mentioned in the introduction, for the case $N>1$ Holmström and Milgrom (1987) present two variants of the continuous-time model that actually do yield optimal incentive schemes that are linear in aggregates, e.g., total profits. One of these involves 
an assumption that the principal observes the time path of total profits rather than the time paths of all the accounts $X_{i}(\cdot)$. We will discuss this approach extensively in the next section. The other one involves an assumption that the agent's effort cost depends only on expected profits rather than the vector of drift rates for the different accounts. In the remainder of this paper we ask what are the discrete-time analogues of these continuoustime results.

In this section we begin with the second variant. The assumption that effort cost depends only on expected profits requires that the cost function in the continuous-time model takes the form

$$
\hat{c}(\mu)=g\left(\sum_{i=1}^{N} \mu_{i}\right)
$$

i.e, the agent's cost depends only on the expected profits he is going to produce: the higher expected profits the more costly it is for the agent. This cost function seems to be very natural, it is easy to deal with, and it has often been used in applications of the Brownian model (see e.g. Holmström and Milgrom (1991) or Itoh (1992)). ${ }^{10}$ It is also close to the specification of Mirrlees (1974), who considers a one-period model where profits are lognormally distributed and the agent chooses the mean of this distribution. Mirrlees assumes that the agent's costs are an increasing and convex function of the mean, i.e., of expected profits. ${ }^{11}$

Using (13) and (7) it is straightforward to show that in the discrete one-period model the cost function corresponding to $(47)$ is given by ${ }^{12}$

$$
c(p)=g(E(p))=g\left(\sum_{i=0}^{N} p_{i} \pi_{i}\right)
$$

In the discrete multi-period model we get

$$
c^{\Delta}\left(p^{\Delta}\right)=\Delta c\left(\hat{p}+\frac{p^{\Delta} \Leftrightarrow \hat{p}}{\Delta^{\frac{1}{2}}}\right)=\Delta g\left(\sum_{i=0}^{N}\left(\hat{p}_{i}+\frac{p_{i}^{\Delta} \Leftrightarrow \hat{p}_{i}}{\Delta^{\frac{1}{2}}}\right) \pi_{i}\right)=\Delta g\left(\frac{E^{\Delta}\left(p^{\Delta}\right)}{\Delta}\right)=\Delta g\left(\underline{\mu}^{\Delta}\right)
$$

\footnotetext{
${ }^{10}$ Furthermore, it admits the interpretation that the "tasks" $\mu_{i}$ are perfect substitutes in the agent's cost function.

${ }^{11}$ Schättler and Sung (1997) use the same specification in a discrete-time model that is used to approximate a continuous-time Brownian-motion model. They run into Mirrlees' problem that the discrete principal-agent problem has no solution.

${ }^{12}$ This specification of the cost function violates Assumption 1 which requires that $c(p)$ is strictly convex. So far, however, strict convexity of $c(\cdot)$ has been used in Proposition 3 only. All of the results of this section hold if $g(\cdot)$ is strictly convex.
} 
where

$$
E^{\Delta}\left(p^{\Delta}\right)=\sum_{i=0}^{N} p_{i}^{\Delta} \pi_{i}^{\Delta}=\Delta \sum_{i=1}^{N} \mu_{i}^{\Delta}
$$

and

$$
\underline{\mu}^{\Delta}=\sum_{i=1}^{N} \mu_{i}^{\Delta}
$$

Finally, let

$$
V^{\Delta}(p)=\sum_{i=0}^{N} p_{i}\left(\pi_{i}^{\Delta} \Leftrightarrow E^{\Delta}(p)\right)^{2}=\Delta \sum_{i=0}^{N} p_{i}\left(\pi_{i} \Leftrightarrow E(p)\right)^{2}
$$

and define

$$
\sigma^{2} \equiv V^{1}(\hat{p})
$$

The following result, which is an immediate corollary of Theorem 1(c), shows that in the limit, as $\Delta \rightarrow 0$, the incentive scheme that implements a given action $\mu$ in the interior of the admissible action space is indeed a linear function of total profits.

Corollary 3 Suppose that in the discrete multi-period model the agent's cost function is given by (49) and that the principal wants to implement a constant action vector $\mu^{\Delta}$, satisfying $0<p_{i}^{\Delta}\left(\mu^{\Delta}\right)<1$ for any sufficiently small $\Delta$, that gives rise to expected profits $\underline{\mu}^{\Delta}$. Let $w$ denote the certainty equivalent of the agent's utility if he does not work for the principal. Assume that as $\Delta$ converges to zero, $\mu^{\Delta}$ converges to $\mu$. Then also the incentive schemes that implement $\mu^{\Delta}$ converge to

$$
s=w+g(\underline{\mu})+g^{\prime} \cdot(\text { total profits } \Leftrightarrow \underline{\mu})+\frac{r}{2}\left(g^{\prime}\right)^{2} \sigma^{2}
$$

which coincides with the incentive scheme that implements $\mu$ in the Brownian model.

At first sight, Corollary 3 seems to provide for the discrete-time approximations of continuous-time models involving the cost specification (47) and the linear incentive scheme (54). However, this interpretation is flawed because in the discrete multi-period models the principal never wants to implement a constant action vector $\mu^{\Delta}$ such that $0<p_{i}\left(\mu^{\Delta}\right)<1$ for all $i$. To see this, go back to the static model with cost specification (48). If the set $P$ of admissible probability vectors is equal to the entire simplex, there 
is nothing to prevent a solution which eliminates practically all risk, and under (48) such a solution will in fact be optimal. Since the effort cost of the agent depends on expected profits only and since total surplus increases when the riskiness of the outcome is reduced, the principal will try to implement any target level of expected profits with as little risk as possible. For this purpose he induces the agent to put all probability mass on the two profit levels just neighboring the target level. If the set $\left(p_{1}, \ldots, p_{N}\right)$ of profit levels in the static model has been specified so that neighboring levels are close to each other, the outcome is "almost" deterministic and the first best can "almost" be implemented. The very core of the agency problem seems to have disappeared.

This result holds in any of the multi-period discrete-time models as well as in the static model. ${ }^{13}$ In each case, the variance of cumulative profits is bounded by the square of the maximum of differences between neighboring profit levels in the static model. In the Brownian motion model, however, the variance is given exogenously and the optimal solution of the principal-agent problem is bounded away from the first-best. Theorem 1 and its Corollaries are not applicable because the sequence of optimal actions in the discrete-time model does not have a well defined limit. For any $i$ for which $p_{i}^{\Delta}=0$, by (7) the corresponding $\mu_{i}^{\Delta}\left(p_{i}^{\Delta}\right)$ is negative and goes out of bounds as $\Delta \rightarrow 0$, whereas for the two indices $i$ on which $p_{i}^{\Delta}$ is concentrated, the corresponding $\mu_{i}^{\Delta}\left(p_{i}^{\Delta}\right)$ is positive (and also goes out of bounds). Not only does the discrete-time version of (48) eliminate the core of the agency problem, but also it gives rise to a discontinuity in the transition from multi-period discrete-time models to continuous time.

The discontinuity in the transition from discrete-time models to continuous time would not be present if the controls $\mu^{\Delta}$ were restricted to a compact set $K$, as in Theorem 2. In this case however the limiting incentive scheme would be given by (44) rather than (54). The Kuhn-Tucker multipliers $\hat{u}_{i}^{*}$ and $\hat{v}_{i}^{*}$ in (44) would depend on $i$ and would in fact prevent the incentive scheme from being a linear function of the profit aggregate. ${ }^{14}$

\footnotetext{
${ }^{13}$ In (49) $\hat{p}$ cancels out of $g(\cdot)$ for any $\Delta$ and the agent's isocost curves are still linear in $p_{i}^{\Delta}$.

${ }^{14}$ In fact, the specification (48) does not fit into the framework of Holmström and Milgrom's discrete time model. If $c(p)$ depends only on the linear aggregate $\sum_{i=0}^{N} p_{i} \pi_{i}$, then the corresponding isocost curves in the probability simplex are linear and there is no natural impediment to the agent's substituting between, say, $p_{i}$ and $p_{j}$. In consequence the principal wants to implement a probability vector on the boundary of the set of admissible vectors. This is incompatible with Assumption A(iv) of Holmström and Milgrom (1987, p. 310) whereby any $p$ on the boundary of the set of admissible probability vectors is prohibitively costly for the agent and hence for the principal.
} 
We conclude that the result that the optimal incentive scheme in the Brownian model is linear in total profits if the agent's cost function depends on expected profits only cannot be approximated by the multi-period discrete-time models.

\section{Approximating Sharing Rules that Are Linear in Aggregates}

In this section we develop a discrete-time analogue of the proposition of Holmström and Milgrom that optimal incentive schemes in the continuous-time model are linear in a given aggregate if the principal observes the time path of this aggregate without observing its individual components. In terms of formal modelling, the assumption is that the principal observes the time path of the accounting aggregate $z(t)=\sum_{i=1}^{N} Z_{i}(t)$ - and hence the time path of total profits - without observing the time paths of the individual accounts $Z_{i}(t)$, $i=1, \ldots, N$. With this assumption, Theorem 8 of Holmström and Milgrom (1987) shows that the optimal incentive scheme is a linear function of the value of this aggregate at $t=1$ (e.g. total profits $z(1) \equiv z$ at the end of the period). This result reflects the basic intuition that nonlinear incentive schemes are vulnerable to manipulation.

Unfortunately, however, Theorem 8 has no immediate discrete-time analogue. To see this, note that in any period $\tau \in\left\{1, \ldots, \frac{1}{\Delta}\right\}$ the principal observes $\sum_{\tau^{\prime}=1}^{\tau} \pi^{\Delta, \tau^{\prime}}$. Since the profit in period $\tau$ is simply $\pi^{\Delta, \tau}=\sum_{\tau^{\prime}=1}^{\tau} \pi^{\Delta, \tau^{\prime}} \Leftrightarrow \sum_{\tau^{\prime}=1}^{\tau-1} \pi^{\Delta, \tau^{\prime}}$, the principal can compute the individual accounts $\tilde{Z}_{i}^{\Delta, \tau}$ even if he observes the time path of total profits only. Since this information can be used to improve the incentive scheme, the principal will use it in the same way as before. Thus, for all $\Delta>0$ we get again linearity in accounts only. In the limit, however, it is impossible to derive the individual accounts $Z_{i}(t)$ from the observation of $z(t)$. Thus, if we want to approximate this result in a discrete framework, we need a stronger form of asymmetric information than is used in Theorem 8 of Holmström and Milgrom. As it turns out, this has a significant impact on the analysis of the discrete-time models themselves.

The discrete models considered in this section have the following structure. The time interval $[0,1]$ is devided in $\frac{1}{\Delta}$ periods each of length $\Delta$. At the beginning of each period $\tau, \tau \in\left\{1, \ldots, \frac{1}{\Delta}\right\}$, the agent observes the past history of profits and chooses his action $\mu^{\Delta, \tau}$. As in Section 3, we embed the discrete model in a continuous model where 
$t \in[0,1]$ and $\mu^{\Delta}(t)=\mu^{\Delta,[t / \Delta]}$. In contrast to the model considered by Holmström and Milgrom, however, we assume that the principal does not observe the time path of profits. Furthermore, we assume that the agent has the possibility to destroy output before he reports total profits $\hat{z}$ to the principal. Thus, the principal observes only the revealed profit $\hat{z} \leq \sum_{i=0}^{N} Z_{i}(1) \equiv z$ at date 1 . Both of these assumptions are required to make sure that the principal cannot construct the accounts $Z_{i}(1)$ for each profit level $i \in\{0, \ldots, N\} .{ }^{15}$

In this model the principal has considerably less information than the agent. However, the principal could ask the agent not only to report final profits but also to report e.g. the time path of profits. ${ }^{16}$ Somewhat more generally, the principal could offer an incentive scheme $S(m)$ which requires the agent to send a message $m$ out of some message space $M(z)$. This message space will, in general, depend on total profits available at date 1 . For example, $M(z)$ could be the set of all possible profit paths with the property that total profits do not exceed $z$. Since the agent can destroy profits unnoticed, it must be the case that $z^{\prime}<z$ implies $M\left(z^{\prime}\right) \subset M(z)$. The following proposition shows that without loss of generality we can restrict attention to incentive schemes that are non-decreasing functions of reported total profits.

Proposition 4 If the principal can implement a control path $\mu^{\Delta}(t)$ with an incentive scheme $S^{\Delta}(m)$, then $\mu^{\Delta}(t)$ can also be implemented with a nondecreasing incentive scheme $s^{\Delta}(z)$, which asks the agent to report total profits $z=\sum_{i=1}^{N} Z_{i}(1)$ truthfully. Furthermore, this can be done so that the utilities of the principal and of the agent are the same under $S^{\Delta}(m)$ and under $s^{\Delta}(z)$.

The proof is a standard application of the revelation principle and is relegated to Appendix A.

Note that if an incentive scheme implements a control path $\mu(\cdot)$ under the information assumptions of Section 2 and if this incentive scheme can be written as a nondecreasing

\footnotetext{
${ }^{15}$ Note that if the agent cannot destroy output the principal may be able to construct these accounts even if he does not observe the time path of profits. To see this, suppose that there are three different profit levels, $\pi \in\left\{-1, \frac{1}{e}, 1\right\}$, where $e$ is Euler's number. In this case the total amount of profits reveals how often each of the three different states occured for any number of periods $\frac{1}{\Delta}$.

${ }^{16}$ Like Holmström and Milgrom (1987) we rule out the possibility that the principal requires the agent to report profits at any points in time $t \in[0,1]$. The idea is that the principal cannot monitor the agent continuously but only at exogenously given discrete points in time. Without this assumption the analysis is considerably more complicated.
} 
function of cumulative profits, then it also implements $\mu(\cdot)$ under the information assumptions in this section: First, the incentive scheme is feasible for the principal because it depends only on total profits. Second, by Proposition 4, the agent will report total profits truthfully since the incentive scheme is nondecreasing. Finally, when the agent chooses the control path $\mu(\cdot)$, he faces exactly the same incentives under the information assumptions of Section 2 and of this section. Hence, if the incentive scheme $\tilde{s}^{\Delta}(\cdot)$ implements $\mu(\cdot)$ under the information assumptions of Section 2 , then the condition that $\tilde{s}^{\Delta}(\cdot)$ can be written as a monotonic function of $z$ is not only necessary, but also sufficient for the implementability of $\mu(\cdot)$ under the information assumptions we impose in this section.

With the condition that final payments to the agent must be given by a nondecreasing function of cumulative total profits, the nature of the multi-period discrete-time agency problem changes dramatically. As a result, the solutions to this problem will no longer exhibit the stationarity properties that were so useful in Corollary 1. To see the issue, note first that in the static agency problem, an incentive scheme involving a nonlinear nondecreasing function of total profits, e.g., a suitable step function, might enable the principal to neutralize at least some of the effects of having to condition on total profits rather than each outcome separately. In a two-period agency problem - and even more so in a general multi-period problem -, his scope for doing so is reduced as the effects of intertemporal aggregation set in and he can, e.g., not distinguish whether a cumulative total profit of two stems from two realizations of one or from one realization of two and one of zero. Even so, his desire to use nonlinearities of the incentive scheme in order to neutralize some of the effects of his inability to condition on individual accounts is not likely to disappear altogether; after all there are limits to intertemporal aggregation, at least as long as the number of periods as well as the set of possible outcomes in each period is finite.

Given an incentive scheme that is a nonlinear function of cumulative total profits, the agent's optimization problem will typically have nonstationary, time-dependent and history-dependent solutions. To see why, suppose that the incentive scheme is a step function. If the horizon is far away, the agent's effort choice will mainly depend on considerations of "global steepness of the staircase", i.e., on the sort of "global" tradeoff between effort and incentive payments that is relevant when he can envisage himself as 
taking the same action repeatedly and wondering what is the impact of the corresponding movement in cumulative total profits on his own income. In contrast, if the agent is near to the end of his horizon, he is more concerned about the local properties of the incentive scheme, e.g., how far away he is from the nearest points of increase or decrease, i.e. the "nearest steps of the staircase" - and also how large these steps are.

These considerations suggest that under the information assumptions made here the multi-period agency problem is rather more difficult to study than in the setting of Section 2 where the solution of the problem can be taken to be stationary. At the same time they suggest that the additional difficulties may perhaps be relatively unimportant when there are many periods and for "most" periods the horizon may be taken to be distant. In this case considerations of intertemporal aggregation would seem to vitiate any attempt of the principal to use nonlinearities of the incentive scheme in order to neutralize the effects of his inability to condition on individual accounts.

The following analysis shows that this is indeed the case. In Theorem 3 below, we show, roughly, that when incentive schemes are restricted to nondecreasing functions of cumulative total profits, a suitably chosen linear incentive scheme is approximately optimal if the number of periods $T=1 / \Delta$ in the multi-period model is large. This provides one analogue of the optimality of linear incentive schemes in continuous time that is established in Theorem 8 of Holmström and Milgrom. The stronger analogue that optimal incentive schemes in the $T$-period problem are approximately linear when $\mathrm{T}$ is large can also be established. However this requires considerably more mathematics and is presented elsewhere (see Hellwig (1998b)).

In this section we assume again that for all $\Delta=1, \frac{1}{2}, \ldots$, the vector $\mu^{\Delta}$ must be chosen from a product $K=\prod_{i=1}^{N}\left[\underline{m}_{i}, M_{i}\right]$ of compact intervals, where $\underline{m}_{i}<0<M_{i}$. Combined with Assumption 1, this implies that $\hat{c}(\cdot)$ is continuously differentiable on the interior of $K$ with uniformly bounded first derivatives. Holmström and Milgrom (1987) and Schättler and Sung (1993) assume in addition that the control paths chosen by the agent lie in the interior of a compact set. Note that the following theorem does not require this assumption. 
Theorem 3 In addition to Assumption 1, suppose that controls are constrained to the compact set $K=\prod_{i=1}^{N}\left[\underline{m}_{i}, M_{i}\right]$. Consider a sequence of discrete models with period length $\Delta, \Delta=1, \frac{1}{2}, \ldots$ There exists an incentive scheme $s^{*}(z)$ which is linear in total profits such that for any $\epsilon>0$ there exists $a \triangleq>0$ with the property that for all $\Delta<\Delta$ the principal's utility loss from using the linear contract $s^{*}(z)$ rather than the optimal contract $s^{\Delta, *}(z)$ is smaller than $\epsilon$. Furthermore, $s^{*}(z)$ is an optimal incentive scheme in the continuous model in which the principal observes only the time path of aggregate profits $z(t)$.

Theorem 3 shows that there exists a discrete-time analoque to Holmström and Milgrom's main result, which says that the optimal incentive scheme in the continuous-time model is linear in aggregates if the principal observes the time path of these aggregates only. In our discrete time model there is not only aggregation accross accounts, but also aggregation over time, since the principal is assumed to only observe the sum of total profits at the end of the last period. Note, however, that there is some implicit aggregation over time in Holmström and Milgrom as well. The principal observes the time path of a Brownian motion. However, at any point in time the incremental change " $d B$ " of this stochastic process is only defined by the integral of the stochastic process between two (arbitrarily close) points in time. But this means that the Holmström-Milgrom assumption whereby the principal observes only the aggregate process $\sum_{i=1}^{N} d B_{i}$ involves some implicit aggregation over time as well.

In the introduction of their paper, Holmström and Milgrom argued that nonlinearities in incentive schemes are vulnerable to manipulation by the agent. However, in most of their actual analysis "manipulation" by the agent does not play any role. Manipulation enters their analysis only in the Brownian model of Theorem 8. If the principal observes the time path of total profits only, then the agent cannot be prevented from choosing a control path that yields a given time path of expected profits at least cost to the agent. This cost minimization leads to marginal costs of raising profits being the same for all accounts so that the term $\sum_{i=1}^{N} \hat{c}_{i} X_{i}(1)$ in the expression for final incentive payments can in fact be written as $\hat{c}_{1} \sum_{i=1}^{N} X_{i}(t)$, a constant times the deviation of cumulative total profits from their mean. 
Our discrete-time analysis shows that there has to be some aggregation over time in addition to the aggregation accross accounts. Furthermore, in our model the agent can destroy profits unnoticed. Thus, the agent has a lot of discretion in how to allocate his effort over time and what to report to the principal. The ability to destroy profits is important because it restricts the principal to nondecreasing incentive schemes. This monotonicity of incentive schemes is important for the claim in the proof of Theorem 3 that as $\Delta$ goes to zero the set of incentive schemes satisfying (A.44) and (MS) shrinks to the set of schemes satisfying (A.45) and (MS). Without monotonicity, one might not be able to rule out sequences of incentive schemes satisfying (A.44), but fluctuating ever more wildly as the range of possible outcome becomes denser and denser and tends towards filling the whole space.

As discussed in the introduction to this section, as long as $\Delta$ is positive a linear contract is unlikely to be optimal in the discrete-time model. However our result shows that it is at least approximately optimal. A linear contract gives a constant incentive pressure over time so that the agent will not exploit nonlinearities of the contract by making his effort depend on past profit realizations. Moreover a linear contract is not vulnerable to manipulation arising from the principal's inability to observe anything other than the cumulative total profit that the agent has chosen not to destroy. These two considerations put a bound on any advantage that a nonliner contract might have over the optimal linear one; moreover this bound is close to zero whenever $\Delta$ is close to zero. 


\section{Appendix A}

Proof of Proposition 1: If the agent chooses $\mu^{\Delta, \tau}=\left(\mu_{1}^{\Delta, \tau}, \ldots, \mu_{N}^{\Delta, \tau}\right)$ in periods $\tau=1, \ldots, \frac{1}{\Delta}$, expected gross profits are

$$
\begin{aligned}
\sum_{\tau=1}^{1 / \Delta} \sum_{i=0}^{N} p_{i}^{\Delta}\left(\mu^{\Delta, \tau}\right) \pi_{i}^{\Delta} & =\sum_{\tau=1}^{1 / \Delta}\left[\left(\hat{p}_{0} \Leftrightarrow \sum_{i=1}^{N} \mu_{i}^{\Delta, \tau} \frac{\Delta^{\frac{1}{2}}}{k_{i}}\right) \pi_{0} \Delta^{\frac{1}{2}}+\sum_{i=1}^{N}\left(\hat{p}_{i}+\mu_{i}^{\Delta, \tau} \frac{\Delta^{\frac{1}{2}}}{k_{i}}\right) \pi_{i} \Delta^{\frac{1}{2}}\right] \\
& =\sum_{\tau=1}^{1 / \Delta}\left[\sum_{i=0}^{N} \hat{p}_{i} \pi_{i} \Delta^{\frac{1}{2}} \Leftrightarrow \sum_{i=1}^{N} \mu_{i}^{\Delta, \tau} \frac{\Delta \pi_{0}}{k_{i}}+\sum_{i=1}^{N} \mu_{i}^{\Delta, \tau} \frac{\Delta \pi_{i}}{k_{i}}\right] \\
& =\sum_{\tau=1}^{1 / \Delta} \sum_{i=1}^{N} \mu_{i}^{\Delta, \tau} \frac{\pi_{i} \Leftrightarrow \pi_{0}}{k_{i}} \Delta \\
& =\Delta \sum_{\tau=1}^{1 / \Delta} \sum_{i=1}^{N} \mu_{i}^{\Delta, \tau}
\end{aligned}
$$

Furthermore, we have

$$
\sum_{\tau=1}^{1 / \Delta} c^{\Delta}\left(p^{\Delta}\left(\mu^{\Delta, \tau}\right)\right)=\sum_{\tau=1}^{1 / \Delta} \Delta c\left(\hat{p}+\frac{p^{\Delta}\left(\mu^{\Delta, \tau}\right) \Leftrightarrow \hat{p}}{\Delta \frac{1}{2}}\right)=\sum_{\tau=1}^{1 / \Delta} \Delta \hat{c}\left(\mu^{\Delta, \tau}\right) .
$$

Obviously, if $\mu^{\Delta, \tau}=\mu$ for all $\tau=1, \ldots, \frac{1}{\Delta}$ and all $\Delta=1, \frac{1}{2}, \ldots$, we have

$$
\sum_{\tau=1}^{1 / \Delta} \sum_{i=0}^{N} p_{i}^{\Delta}(\mu) \pi_{i}^{\Delta}=\frac{1}{\Delta} \sum_{i=1}^{N} \mu_{i} \Delta=\sum_{i=1}^{N} \mu_{i}
$$

and

$$
\sum_{\tau=1}^{1 / \Delta} c^{\Delta}(p(\mu))=\frac{1}{\Delta} \Delta \hat{c}(\mu)=\hat{c}(\mu) .
$$

Thus, in this case total expected profits and total costs to the agent depend on $\mu$ but are independent of the length of each subperiod $\Delta$.

Q.E.D.

Proof of Theorem 1:

(a) Consider any time interval $\left[t, t^{\prime}\right], t^{\prime}>t$. Suppose that the agent takes action $\mu^{\Delta, \tau}$ in period $\tau \in\left\{[t / \Delta]+1,[t / \Delta]+2, \ldots,\left[t^{\prime} / \Delta\right]\right\}$. Then, in each of these periods the probability that $\tilde{A}_{i}^{\Delta, \tau}=1$ equals $p_{i}^{\Delta}\left(\mu^{\Delta, \tau}\right)$ which is defined by (9) and (10) above. Furthermore, since the time path of actions $\left\{\mu^{\Delta, \tau}\right\}$ is deterministic, any $\tilde{A}_{i}^{\Delta, \tau_{1}}$ and $\tilde{A}_{i}^{\Delta, \tau_{2}}$ are stochastically independent for any $\tau_{1}, \tau_{2} \in\left\{[t / \Delta]+1,[t / \Delta]+2, \ldots,\left[t^{\prime} / \Delta\right]\right\}$, 
$\tau_{1} \neq \tau_{2}$. The distribution of $X_{i}^{\Delta}\left(t^{\prime}\right) \Leftrightarrow X_{i}^{\Delta}(t)$ is characterized by:

$$
\begin{aligned}
\mathrm{E}\left(X_{i}^{\Delta}\left(t^{\prime}\right) \Leftrightarrow X_{i}^{\Delta}(t)\right) & =\mathrm{E}\left(\tilde{X}_{i}^{\Delta}\left(\left[\frac{t^{\prime}}{\Delta}\right]\right) \Leftrightarrow \tilde{X}_{i}^{\Delta}\left(\left[\frac{t}{\Delta}\right]\right)\right) \\
& =k_{i} \Delta^{\frac{1}{2}} \sum_{\tau=t / \Delta+1}^{t^{\prime} / \Delta}\left(p_{i}^{\Delta}\left(\mu^{\Delta, \tau}\right) \Leftrightarrow p_{i}^{\Delta}\left(\mu^{\Delta, \tau}\right)\right)=0
\end{aligned}
$$

$\operatorname{Var} \quad\left(X_{i}^{\Delta}\left(t^{\prime}\right) \Leftrightarrow X_{i}^{\Delta}(t)\right)=\operatorname{Var}\left(\tilde{X}_{i}^{\Delta}\left(\left[\frac{t^{\prime}}{\Delta}\right]\right) \Leftrightarrow \tilde{X}_{i}^{\Delta}\left(\left[\frac{t}{\Delta}\right]\right)\right)$

$$
\begin{aligned}
& =k_{i}^{2} \Delta \sum_{\tau=t / \Delta+1}^{t^{\prime} / \Delta} p_{i}^{\Delta}\left(\mu^{\Delta, \tau}\right)\left(1 \Leftrightarrow p_{i}^{\Delta}\left(\mu^{\Delta, \tau}\right)\right) \\
& =k_{i}^{2} \Delta \sum_{\tau=t / \Delta+1}^{t^{\prime} / \Delta}\left(\hat{p}_{i}+\mu_{i}^{\Delta, \tau} \frac{\Delta^{\frac{1}{2}}}{k_{i}}\right)\left(1 \Leftrightarrow \hat{p}_{i} \Leftrightarrow \mu_{i}^{\Delta, \tau} \frac{\Delta^{\frac{1}{2}}}{k_{i}}\right) \\
& =k_{i}^{2} \Delta \sum_{\tau=t / \Delta+1}^{t^{\prime} / \Delta}\left(\hat{p}_{i} \Leftrightarrow \hat{p}_{i}^{2} \Leftrightarrow \hat{p}_{i} \mu_{i}^{\Delta, \tau} \frac{\Delta^{\frac{1}{2}}}{k_{i}}+\mu_{i}^{\Delta, \tau} \frac{\Delta^{\frac{1}{2}}}{k_{i}} \Leftrightarrow \hat{p}_{i} \mu_{i}^{\Delta, \tau} \frac{\Delta^{\frac{1}{2}}}{k_{i}} \Leftrightarrow\left(\mu_{i}^{\Delta, \tau}\right)^{2} \frac{\Delta}{k_{i}^{2}}\right) \\
& =k_{i}^{2}\left(t^{\prime} \Leftrightarrow t\right) \hat{p}_{i}\left(1 \Leftrightarrow \hat{p}_{i}\right)+O(\Delta)
\end{aligned}
$$

$$
\begin{aligned}
\operatorname{Cov} & \left(X_{i}^{\Delta}\left(t^{\prime}\right) \Leftrightarrow X_{i}^{\Delta}(t), X_{j}^{\Delta}\left(t^{\prime}\right) \Leftrightarrow X_{j}^{\Delta}(t)\right) \\
& =\operatorname{Cov}\left(\tilde{X}_{i}^{\Delta}\left(\left[\frac{t^{\prime}}{\Delta}\right]\right) \Leftrightarrow \tilde{X}_{i}^{\Delta}\left(\left[\frac{t}{\Delta}\right]\right), \tilde{X}_{j}^{\Delta}\left(\left[\frac{t^{\prime}}{\Delta}\right]\right) \Leftrightarrow \tilde{X}_{j}^{\Delta}\left(\left[\frac{t}{\Delta}\right]\right)\right) \\
& =k_{i} k_{j} \Delta \sum_{\tau=t / \Delta+1}^{t^{\prime} / \Delta} p_{i}^{\Delta}\left(\mu^{\Delta, \tau}\right) p_{j}^{\Delta}\left(\mu^{\Delta, \tau}\right) \\
& =k_{i} k_{j} \Delta \sum_{\tau=t / \Delta+1}^{t^{\prime} / \Delta}\left(\hat{p}_{i}+\mu_{i}^{\Delta, \tau} \frac{\Delta^{\frac{1}{2}}}{k_{i}}\right)\left(\hat{p}_{j}+\mu_{j}^{\Delta, \tau} \frac{\Delta^{\frac{1}{2}}}{k_{j}}\right) \\
& =k_{i} k_{j} \Delta \sum_{\tau=t / \Delta+1}^{t^{\prime} / \Delta}\left(\hat{p}_{i} \hat{p}_{j}+\hat{p}_{i} \mu_{j}^{\Delta, \tau} \frac{\Delta^{\frac{1}{2}}}{k_{j}}+\hat{p}_{j} \mu_{i}^{\Delta, \tau} \frac{\Delta^{\frac{1}{2}}}{k_{i}}+\mu_{i}^{\Delta, \tau} \mu_{j}^{\Delta, \tau} \frac{\Delta}{k_{i} k_{j}}\right) \\
& =\Leftrightarrow k_{i} k_{j} \hat{p}_{i} \hat{p}_{j}\left(t^{\prime} \Leftrightarrow t\right)+O(\Delta)
\end{aligned}
$$

where $O(\Delta)$ contains all terms that vanish as $\Delta$ goes to 0 . Thus, in the limit as $\Delta$ goes to zero, the variances and covariances are independent of the time path of actions $\left\{\mu^{\Delta, \tau}\right\}$.

The stochastic process $X^{\Delta}(t)$ satisfies the conditions of Prohorov's generalization of

Donsker's Theorem. ${ }^{17}$ In particular, note that the maximum of profits that can be

${ }^{17}$ See Billingsley (1968), Theorem 10.1 in conjunction with Problem 10.1 (p. 77) and Problem 16.7 (p. 143). 
obtained in any period is given by $\pi_{N}^{\Delta}=\pi_{N} \Delta^{\frac{1}{2}}$ and goes to zero as $\Delta$ goes to zero which implies that the Lindeberg condition is satisfied. Hence, as $\Delta \rightarrow 0, X^{\Delta}(t)$ converges in distribution to a multidimensional Brownian motion with drift 0 and covariance matrix $\Sigma$.

(b) Suppose the agent takes the time path of actions $\mu^{\Delta, \tau}, \tau=1, \ldots, \frac{1}{\Delta}$, where $\mu^{\Delta}(t)$ converges to $\mu(t)$ uniformly in $t$ as $\Delta$ goes to zero. Since $\mu(t)$ is continuous, there exists a compact set $K \subset I^{N}$ such that for any sufficiently small $\Delta$ one has $\mu^{\Delta}(t) \in$ $K$ for all $t$. Since $\hat{c}(\cdot)$ is continuous - and hence bounded on $K$ - we find for the agent's cost in the limit as $\Delta$ goes to 0 :

$$
\lim _{\Delta \rightarrow 0} \sum_{\tau=1}^{1 / \Delta} \Delta \hat{c}\left(\mu^{\Delta, \tau}\right)=\lim _{\Delta \rightarrow 0} \int_{\Delta}^{1} \hat{c}\left(\mu^{\Delta}(t)\right) d t=\int_{0}^{1} \hat{c}(\mu(t)) d t
$$

(c) Substituting (19) in (18) yields:

$$
\begin{aligned}
\sum_{\tau=1}^{1 / \Delta} \tilde{s}^{\Delta, \tau}=\sum_{\tau=1}^{\Delta} \Delta \hat{c}\left(\mu^{\Delta, \tau}\right)+\sum_{\tau=1}^{1 / \Delta} \sum_{i=1}^{N} \hat{c}_{i}\left(\mu^{\Delta, \tau}\right) \tilde{X}_{i}^{\Delta, \tau} \\
+\frac{r}{2} \sum_{\tau=1}^{1 / \Delta} \sum_{i=0}^{N} \Delta \tilde{A}_{i}^{\Delta, \tau}\left[\hat{c}_{i}\left(\mu^{\Delta, \tau}\right) k_{i} \Leftrightarrow \sum_{j=1}^{N} \hat{p}_{j} \hat{c}_{j}\left(\mu^{\Delta, \tau}\right) k_{j}\right]^{2}+O\left(\Delta^{\frac{1}{2}}\right)
\end{aligned}
$$

From (b), the first term in (A.11) converges to $\int \hat{c}(\mu(t)) d t$ as $\Delta$ converges to zero. As for the second term, the same argument as in (b) implies that for any sufficiently small $\Delta, \mu^{\Delta, \tau}$ belongs to a compact set $K$ for all $\tau$, and $\hat{c}_{i}\left(\mu^{\Delta, \tau}\right)$ is bounded, uniformly in $\tau$. From part (a) therefore, one finds that for any sufficiently small $\Delta^{\prime}$, the sums

$$
\sum_{\tau=1}^{1 / \Delta} \hat{c}_{i}\left(\mu^{\Delta^{\prime},\left[\tau \Delta / \Delta^{\prime}\right]}\right) \tilde{X}_{i}^{\Delta, \tau}=\sum_{\tau=1}^{1 / \Delta} \hat{c}_{i}\left(\mu^{\Delta^{\prime}, \tau}\right)\left[X_{i}^{\Delta}\left(\tau \Delta^{\prime}\right) \Leftrightarrow X_{i}^{\Delta}\left(\tau \Delta^{\prime} \Leftrightarrow \Delta^{\prime}\right)\right]
$$

converge in distribution to

$$
\sum_{\tau=1}^{1 / \Delta} \hat{c}_{i}\left(\mu^{\Delta^{\prime}, \tau}\right)\left[X_{i}^{\Delta}\left(\tau \Delta^{\prime}\right) \Leftrightarrow X_{i}^{\Delta}\left(\tau \Delta^{\prime} \Leftrightarrow \Delta^{\prime}\right)\right]=\int_{0}^{1} \hat{c}_{i}\left(\mu^{\Delta^{\prime}}(t)\right) d X_{i}(t),
$$

as $\Delta$ goes to zero, uniformly in $\Delta^{\prime}$. One also has $\lim _{\Delta^{\prime} \rightarrow 0} \hat{c}_{i}\left(\mu^{\Delta^{\prime}}(t)\right)=\hat{c}_{i}(\mu(t))$ uniformly in $t$, and hence $\operatorname{plim}_{\Delta^{\prime} \rightarrow 0} \int \hat{c}_{i}\left(\mu^{\Delta^{\prime}}(t)\right) d X_{i}(t)=\int \hat{c}_{i}(\mu(t)) d X_{i}(t)$. Therefore the sums $\sum_{\tau} \hat{c}_{i}\left(\mu^{\Delta, \tau}\right) X_{i}^{\Delta, \tau}$ in the second term of (A.11) converge in distribution to the stochastic integral $\int \hat{c}_{i}(\mu(t)) d X_{i}(t)$ as $\Delta$ goes to zero. 
Turning to the third term in (A.11), we rewrite this in the form

$$
\begin{array}{r}
\frac{r}{2} \sum_{\tau=1}^{1 / \Delta} \sum_{i=0}^{N} \Delta \quad\left(\tilde{A}_{i}^{\Delta, \tau} \Leftrightarrow p_{i}^{\Delta}\left(\mu^{\Delta, \tau}\right)\right)\left[\hat{c}_{i}\left(\mu^{\Delta, \tau}\right) k_{i} \Leftrightarrow \sum_{j=1}^{N} \hat{p}_{j} \hat{c}_{j}\left(\mu^{\Delta, \tau}\right) k_{j}\right]^{2} \\
+\frac{r}{2} \sum_{\tau=1}^{1 / \Delta} \sum_{i=0}^{N} p_{i}^{\Delta}\left(\mu^{\Delta, \tau}\right)\left[\hat{c}_{i}\left(\mu^{\Delta, \tau}\right) k_{i} \Leftrightarrow \sum_{j=1}^{N} \hat{p}_{j} \hat{c}_{j}\left(\mu^{\Delta, \tau}\right) k_{j}\right]^{2}
\end{array}
$$

By the law of large numbers, the first of these terms converges to zero almost surely as $\Delta$ goes to zero. The second term converges to the integral $\frac{r}{2} \int_{0}^{1} \hat{c}^{\prime}(\mu(t)) \Sigma\left[c^{\prime}(\mu(t))\right]^{T} d t$. Thus, if the principal wants to implement the time path of actions $\mu(t), t \in[0,1]$, such that the agent's certainty equivalent is $w$, then the incentive scheme that implements $\mu(t)$ converges to

$$
s=w+\int_{0}^{1} \hat{c}(\mu(t)) d t+\int_{0}^{1} \hat{c}^{\prime}(\mu(t)) d X+\frac{r}{2} \int_{0}^{1} \hat{c}^{\prime}(\mu(t)) \Sigma\left[c^{\prime}(\mu(t))\right]^{T} d t .
$$

Q.E.D.

Proof of Proposition 2: To simplify the notation write

$$
a_{i}^{\Delta}:=1 \Leftrightarrow r\left(\hat{c}_{i} \Leftrightarrow \hat{u}_{i}^{\Delta}+\hat{v}_{i}^{\Delta}\right) k_{i} \Delta^{\frac{1}{2}}+\sum_{j=1}^{N} p_{j}^{\Delta}\left(\mu^{\Delta}\right) r\left(\hat{c}_{j} \Leftrightarrow \hat{u}_{j}^{\Delta}+v_{j}^{\Delta}\right) k_{j} \Delta^{\frac{1}{2}}
$$

and note that the first-order conditions for the maximization of (39) with respect to $\hat{u}_{i}^{\Delta}$ and $\hat{v}_{i}^{\Delta}$ under the constraints (38) can be written as:

$$
\begin{aligned}
& \frac{1}{a_{i}^{\Delta}} \leq \sum_{j=1}^{N} \frac{p_{j}^{\Delta}\left(\mu^{\Delta}\right)}{a_{j}^{\Delta}}, \quad \text { with equality if } \hat{u}_{i}^{\Delta}>0, \text { and } \hat{v}_{i}^{\Delta}=0 \text {, if } \mu_{i}^{\Delta}=\underline{m}_{i} ; \\
& \hat{u}_{i}^{\Delta}=\hat{v}_{i}^{\Delta}=0, \text { if } \underline{m}_{i}<\mu_{i}^{\Delta}<M_{i} ; \\
& \frac{1}{a_{i}^{\Delta}} \geq \sum_{j=1}^{N} \frac{p_{j}^{\Delta}\left(\mu^{\Delta}\right)}{a_{j}^{\Delta}}, \quad \text { with equality if } \hat{v}_{i}^{\Delta}>0, \text { and } \hat{u}_{i}^{\Delta}=0 \text {, if } \mu_{i}^{\Delta}=M_{i} ;
\end{aligned}
$$

Suppose first that $\hat{u}_{i}^{\Delta}>0$ and $\hat{v}_{j}^{\Delta}>0$ for some $i$ and $j$. Then (A.17) and (A.19) imply $a_{i}^{\Delta}=a_{j}^{\Delta}$ as well as $\hat{v}_{i}^{\Delta}=\hat{u}_{j}^{\Delta}=0$, and one immediately obtains $k_{i} \hat{u}_{i}^{\Delta}+k_{j} \hat{v}_{j}^{\Delta}=$ $k_{i} \hat{c}_{i}\left(\mu^{\Delta}\right) \Leftrightarrow k_{j} \hat{c}_{j}\left(\mu^{\Delta}\right) \leq \gamma\left(\mu^{\Delta}\right)$. Suppose next that $\hat{u}_{i}^{\Delta}=0$ for all $i$ and $\hat{v}_{i}^{\Delta}>0$ for some $i$. Let $J$ be the set of indices $j$ for which $\hat{v}_{j}^{\Delta}=0$ and note that $0 \in J$ so $J \neq \phi$. Let $M:=\sum_{j \in J} \frac{p_{j}^{\Delta}\left(\mu^{\Delta}\right)}{a_{j}^{\Delta}}$ and note that (A.19) implies $M=\sum_{j \in J} \frac{p_{j}^{\Delta}\left(\mu^{\Delta}\right)}{a_{j}^{\Delta}}+\sum_{j \notin J} p_{j}^{\Delta}\left(\mu^{\Delta}\right) M$, hence $M=\frac{\sum_{j \in J} p_{j}^{\Delta}\left(\mu^{\Delta}\right) / a_{j}^{\Delta}}{\sum_{j \in J} p_{j}^{\Delta}\left(\mu^{\Delta}\right)}$ and $\frac{1}{M} \geq \min _{j \in J} a_{j}^{\Delta}$. For any $i \notin J,\left(\right.$ A.19) implies $a_{i}^{\Delta}=\frac{1}{M}$, hence 
$a_{i}^{\Delta} \geq \min _{j \in J} a_{j}^{\Delta}$. This in turn yields $k_{i} v_{i}^{\Delta} \leq \max _{j \in J} k_{j} \hat{c}_{j}\left(\mu^{\Delta}\right) \Leftrightarrow k_{i} \hat{c}_{i}\left(\mu^{\Delta}\right) \leq \gamma\left(\mu^{\Delta}\right)$. Finally, if $\hat{v}_{i}^{\Delta}=0$ for all $i$ and $\hat{u}_{i}^{\Delta}>0$ for some $i$, a precisely symmetric argument shows that for all $i, k_{i} \hat{u}_{i}^{\Delta} \leq \max _{j \in J} k_{j} \hat{c}_{j}\left(\mu^{\Delta}\right) \Leftrightarrow k_{i} \hat{c}_{i}\left(\mu^{\Delta}\right) \leq \gamma\left(\mu^{\Delta}\right)$, where $J$ now is the set of indices $j$ for which $\hat{u}_{j}^{\Delta}>0$.

Q.E.D.

Proof of Proposition 3: Trivially, any limit $(\mu, \underline{\hat{u}}, \underline{\hat{\hat{v}}})$ of a sequence $\left\{\left(\mu^{\Delta}, \underline{\hat{u}}^{\Delta}, \underline{\hat{v}}^{\Delta}\right)\right\}$ of vectors in $K \times I R_{+}^{N} \times I R_{+}^{N}$ that satisfy (42) as well as (38) for all $\Delta$ will itself belong to the set of vectors in $K \times I R_{+}^{N} \times \mathbb{R}_{+}^{N}$ that satisfy (43). To prove the proposition it therefore suffices to show that any vector $(\mu, \underline{\hat{\hat{u}}}, \underline{\hat{\hat{v}}}) \in K \times I R_{+}^{N} \times I R_{+}^{N}$ that satisfies (43) can in fact be approximated by a sequence of vectors $\left(\mu^{\Delta}, \underline{\hat{x}}^{\Delta}, \underline{\hat{v}}^{\Delta}\right) \in I C(\Delta)$.

Given $(\mu, \underline{\hat{u}}, \underline{\hat{v}}) \in K \times I R_{+}^{N} \times I R_{+}^{N}$ satisfying (43), for any $\Delta>0$, let

$$
s_{i}^{\Delta}=\Delta \hat{c}(\mu) \Leftrightarrow \frac{1}{r} \ln \left[1 \Leftrightarrow r\left(\hat{c}_{i} \Leftrightarrow \hat{u}_{i}+\hat{v}_{i}\right) k_{i} \Delta^{\frac{1}{2}}+r \sum_{j=1}^{N} p_{j}^{\Delta}(\mu)\left(\hat{c}_{j} \Leftrightarrow \hat{u}_{j}+\hat{v}_{j}\right) k_{j} \Delta^{\frac{1}{2}}\right],
$$

and let $\mu^{\Delta}$ be a solution to the problem of maximizing $\Leftrightarrow \sum_{i=1}^{N} p_{i}^{\Delta}\left(\mu^{\Delta}\right) e^{-r\left(s_{i}^{\Delta}-\Delta \hat{c}\left(\mu^{\Delta}\right)\right)}$ over the set $K$. Moreover for $i=1, \ldots, N$, let $r \Delta^{\frac{1}{2}} k_{i} \hat{u}_{i}^{\Delta}, r \Delta^{\frac{1}{2}} k_{i} \hat{v}_{i}^{\Delta}$ be the Kuhn-Tucker multipliers corresponding to the constraints $\mu_{i}^{\Delta} \geq \underline{m}_{i}$ and $M_{i} \geq \mu_{i}^{\Delta}$ in this maximization, and write $\underline{\hat{\hat{x}}}^{\Delta}=\left(\hat{u}_{1}^{\Delta}, \ldots, \hat{u}_{N}^{\Delta}\right), \underline{\hat{v}}^{\Delta}=\left(\hat{v}_{1}^{\Delta}, \ldots, \hat{v}_{N}^{\Delta}\right)$. Then clearly $\left(\mu^{\Delta}, \underline{\hat{u}}^{\Delta}, \underline{\hat{v}}^{\Delta}\right) \in I C(\Delta)$. We also show that $\left(\mu^{\Delta}, \underline{\hat{u}}^{\Delta}, \underline{\hat{v}}^{\Delta}\right)$ converges to $(\mu, \underline{\hat{u}}, \underline{\hat{v}})$ as $\Delta$ converges to zero.

The argument is based on Berge's (1959) maximum theorem. For the given incentive scheme, the maximization problem defining $\mu^{\Delta}$ is equivalent to the problem of minimizing

$$
e^{-r \Delta\left(\hat{c}(\mu)-\hat{c}\left(\mu^{\Delta}\right)\right)}\left[1 \Leftrightarrow r \sum_{i=1}^{N}\left(p_{i}^{\Delta}\left(\mu^{\Delta}\right) \Leftrightarrow p_{i}^{\Delta}(\mu)\left(\hat{c}_{i} \Leftrightarrow \hat{u}_{i}+\hat{v}_{i}\right) k_{i} \Delta^{\frac{1}{2}}\right]\right.
$$

over the set $K$. Given the specification of $p_{i}^{\Delta}(\cdot)$, minimization of (A.21) with respect to $\mu^{\Delta}$ in turn is equivalent to the minimization of

$$
e^{-r \Delta\left(\hat{c}(\mu)-\hat{c}\left(\mu^{\Delta}\right)\right)}\left[1 \Leftrightarrow r \Delta \sum_{i=1}^{N}\left(\mu_{i}^{\Delta} \Leftrightarrow \mu_{i}\right)\left(\hat{c}_{i} \Leftrightarrow \hat{u}_{i}+\hat{v}_{i}\right)\right]
$$

with respect to $\mu^{\Delta}$. Upon subtracting the constant 1 and dividing by $\Delta$, one finds that given the minimization of (A.22) is equivalent to the minimization of

$$
\frac{e^{-r \Delta\left(\hat{c}(\mu)-\hat{c}\left(\mu^{\Delta}\right)\right)} \Leftrightarrow 1}{\Delta} \Leftrightarrow r \sum_{i=1}^{N}\left(\mu_{i}^{\Delta} \Leftrightarrow \mu_{i}\right)\left(\hat{c}_{i} \Leftrightarrow \hat{u}_{i}+\hat{v}_{i}\right) e^{-r \Delta\left(\hat{c}(\mu)-\hat{c}\left(\mu^{\Delta}\right)\right)} .
$$


As $\Delta$ goes to zero and $\mu^{\Delta}$ converges to say $\mu^{*}$, the minimand in (A.23) converges to:

$$
r\left(\hat{c}\left(\mu^{*}\right) \Leftrightarrow \hat{c}(\mu)\right) \Leftrightarrow r \sum_{i=1}^{N}\left(\mu_{i}^{*} \Leftrightarrow \mu_{i}\right)\left(\hat{c}_{i}(\mu) \Leftrightarrow \hat{u}_{i}+\hat{v}_{i}\right) .
$$

By Berge's maximum theorem it follows that any limit of a subsequence of minimizers of (A.23) must itself be a minimizer of (A.24). Since $\hat{c}(\cdot)$ is strictly concave, (A.24) has a unique minimum at $\mu^{*}=\mu$. Any convergent subsequence of minimizers of (A.23) must therefore converge to $\mu$. Since $K$ is compact and all convergent subsequences have the same limit, it follows that the sequence $\left\{\mu^{\Delta}\right\}$ converges to $\mu$. Convergence of the associated vectors of normalized Kuhn-Tucker mulitipliers $\underline{\hat{u}}^{\Delta}, \underline{\hat{v}}^{\Delta}$ to $\underline{\hat{u}}, \underline{\hat{v}}$ follows by taking limits in the Kuhn-Tucker conditions for $\mu^{\Delta}$. This shows that the given $(\mu, \underline{\hat{u}}, \underline{\hat{v}}) \in$ $K \times I R_{+}^{N} \times I R_{+}^{N}$ satisfying (43) can in fact be approximated by a sequence of vectors $\left(\mu^{\Delta}, \underline{\hat{u}}^{\Delta}, \underline{\hat{v}}^{\Delta}\right) \in I C(\Delta)$.

Q.E.D.

Proof of Theorem 2: By Proposition C.1 and Remark C.1 in Appendix C, a constant control path $\mu$ is implementable by an incentive scheme $s(\cdot)$ in the continuous-time model if and only if there exist vectors $\underline{\hat{u}}=\left(\hat{u}_{1}, \ldots, \hat{u}_{N}\right), \underline{\hat{v}}=\left(\hat{v}_{1}, \ldots, \hat{v}_{N}\right)$ such that $\mu$ and $\underline{\hat{u}}, \underline{\hat{v}}$ satisfy the complementary slackness condition (38), and moreover $s(\cdot)$ has the representation:

$$
s(X)=w+\hat{c}(\mu)+\sum_{i=1}^{N}\left(\hat{c}_{i}(\mu) \Leftrightarrow \hat{u}_{i}+\hat{v}_{i}\right) X_{i}(1)+\frac{r}{2} \sum_{i=1}^{N} \sum_{j=1}^{N}\left(\hat{c}_{i} \Leftrightarrow \hat{u}_{i}+\hat{v}_{i}\right) \sigma_{i j}\left(\hat{c}_{i} \Leftrightarrow \hat{u}_{i}+v_{i}\right),
$$

The principal's expected payoff from using the scheme $s(\cdot)$ to implement the constant control path $\mu$ is therefore computed as

$$
U^{P}(\mu, \underline{\hat{u}}, \underline{\hat{v}})=\sum_{i=1}^{N} \mu_{i} \Leftrightarrow w \Leftrightarrow \hat{c}(\mu) \Leftrightarrow \frac{r}{2} \sum_{i=1}^{N} \sum_{j=1}^{N}\left(\hat{c}_{i} \Leftrightarrow \hat{u}_{i}+\hat{v}_{i}\right) \sigma_{i j}\left(\hat{c}_{i} \Leftrightarrow \hat{u}_{i}+v_{i}\right),
$$

Therefore a constant control path $\mu$ and incentive scheme $s(\cdot)$ implementing $\mu$ are optimal for the principal in the continuous-time model if and only if $\mu$ and the associated vectors $\hat{u}$ and $\hat{v}$ maximize (A.26) subject to the complementary slackness conditions (43).

Now consider the transition from the multiperiod discrete-time models to the continuoustime model. Expanding the logarithmic term in (40) in a Taylor series and taking limits as $\Delta$ goes to zero we obtain

$$
\lim _{\Delta \rightarrow 0} \quad U^{P \Delta}\left(\mu^{\Delta}, \underline{\hat{u}}^{\Delta}, \underline{\hat{v}}^{\Delta}\right)
$$




$$
\begin{aligned}
& =\sum_{i=1}^{N} \mu_{i} \Leftrightarrow w \Leftrightarrow \hat{c}(\mu) \Leftrightarrow \frac{r}{2} \sum_{i=1}^{N} \hat{p}_{i}\left[\left(\hat{c}_{i} \Leftrightarrow \hat{u}_{i}+\hat{v}_{i}\right) k_{i} \Leftrightarrow \sum_{j=1}^{N} \hat{p}_{j}\left(\hat{c}_{j} \Leftrightarrow \hat{u}_{j}+v_{j}\right) k_{j}\right]^{2} \\
& =U^{P}(\mu, \underline{\hat{u}}, \underline{\hat{v}})
\end{aligned}
$$

for any sequence $\left\{\mu^{\Delta}, \underline{\hat{u}}^{\Delta}, \underline{\hat{v}}^{\Delta}\right\}$ that converges to a limit $(\mu, \underline{\hat{u}}, \underline{\hat{v}}) \in K \times I R_{+}^{N} \times I R_{+}^{N}$ as $\Delta$ goes to zero. In view of (A.27) and Proposition 3, Berge's maximum theorem implies that if the sequence $\left\{\mu^{\Delta *}, \hat{u}_{1}^{\Delta *}, \hat{v}_{1}^{\Delta *}, \ldots, \hat{u}_{N}^{\Delta *}, \hat{v}_{N}^{\Delta *}\right\}$ of constant optimal control paths and associated Kuhn-Tucker multipliers in the discrete-time problems has a subsequence which converges to a limit $\left(\mu^{*}, \hat{u}_{1}^{*}, \hat{v}_{1}^{*}, \ldots, \hat{u}_{N}^{*}, \hat{v}_{N}^{*}\right)$, then $\left(\mu^{*}, \hat{u}_{1}^{*}, \hat{v}_{1}^{*}, \ldots, \hat{u}_{N}^{*}, \hat{v}_{N}^{*}\right)$ maximizes $U^{P}(\mu, \underline{\hat{u}}, \underline{\hat{v}})$ subject to the constraint (43) so $\mu^{*}$ and the incentive scheme $s^{*}(\cdot)$ given by (44) are optimal for the principal in the continuous-time problem.

It remains to be shown that the sequence $\left\{\mu^{\Delta *}, \hat{u}_{1}^{\Delta *}, \hat{v}_{1}^{\Delta *}, \ldots, \hat{u}_{N}^{\Delta *}, \hat{v}_{N}^{\Delta *}\right\}$ actually has a convergent subsequence. Since $\mu^{\Delta *} \in K$ for all $\Delta$, certainly the sequence $\left\{\mu^{\Delta *}\right\}$ has a subsequence which converges to a limit $\mu^{*}$. To economize on notation, we identify the convergent subsequence with the original sequence. For each $\Delta$, let $\underline{\hat{u}}^{\Delta}=\left(\hat{u}_{1}^{\Delta}, \ldots, \hat{u}_{N}^{\Delta}\right)$, $\underline{\hat{v}}^{\Delta}=\left(\hat{v}_{1}^{\Delta}, \ldots, \hat{v}_{N}^{\Delta}\right)$ be the solution to the principal's "relaxed problem" of maximizing $U^{P \Delta}\left(\mu^{*}, \underline{\hat{u}}, \underline{\hat{v}}\right)$ with respect to $\underline{\hat{u}}$ and $\underline{\hat{v}}$ subject only to the complementary slackness conditions (38). By Proposition 2, we have $\hat{u}_{i}^{\Delta} \leq \frac{\gamma\left(\mu^{\Delta *}\right)}{k_{i}}$ and $\hat{v}_{i}^{\Delta} \leq \frac{\gamma\left(\mu^{\Delta *}\right)}{k_{i}}$ for all $i$ and all $\Delta$, hence $\hat{u}_{i}^{\Delta} \leq \frac{\hat{\gamma}}{k_{i}}$ and $\hat{v}_{i}^{\Delta} \leq \frac{\hat{\gamma}}{k_{i}}$ for all $i$ and all $\Delta$. The sequence $\left\{\underline{\hat{u}}^{\Delta}, \underline{\hat{v}}^{\Delta}\right\}$ must therefore have a convergent subsequence. Moreover another application of Berge's maximum theorem shows that any limit $\left(\underline{\hat{u}}^{*}, \underline{\hat{v}}^{*}\right)$ of a subsequence of $\left\{\underline{\hat{u}}^{\Delta}, \underline{\hat{v}}^{\Delta}\right\}$ must be maximizing $U^{P}\left(\mu^{*}, \underline{\hat{u}}, \underline{\hat{v}}\right)$ over the set of $(\underline{\hat{u}}, \underline{\hat{v}})$ satisfying $(43)$. Given that the function $U^{P}\left(\mu^{*}, \cdot, \cdot\right)$ is strictly concave, the latter maximizer is unique, so the limit $\left(\underline{\hat{u}}^{*}, \underline{\hat{v}}^{*}\right)$ must be the same for all convergent subsequences of $\left\{\underline{\hat{u}}^{\Delta}, \underline{\hat{v}}^{\Delta}\right\}$, and the sequence $\left\{\underline{\hat{\hat{u}}}^{\Delta}, \underline{\hat{v}}^{\Delta}\right\}$ itself must be converging to $\left(\underline{\hat{u}}^{*}, \underline{\hat{v}}^{*}\right)$.

We claim that the "true" vectors of Kuhn-Tucker multipliers $\underline{\hat{u}}^{\Delta *}$ and $\underline{\hat{v}}^{\Delta *}$ must also be converging to $\underline{\hat{u}}^{*}$ and $\underline{\hat{v}}^{*}$, so $\left(\mu^{*}, \underline{\hat{u}}^{*}, \underline{\hat{v}}^{*}\right)$ is indeed a limit point of the sequence $\left\{\mu^{\Delta *}, \hat{u}_{1}^{\Delta *}, \hat{v}_{1}^{\Delta *}, \ldots, \hat{u}_{N}^{\Delta *}, \hat{v}_{N}^{\Delta *}\right\}$. Let $U^{P *}$ be the maximum value of $U^{P}(\cdot, \cdot, \cdot)$ subject to the constraint (43). In view of (A.27) and the definition of $\left(\underline{\hat{u}}^{\Delta}\right.$ and $\underline{\hat{v}}^{\Delta}$, we have:

$$
\begin{aligned}
\lim \sup _{\Delta \rightarrow 0} U^{P \Delta}\left(\mu^{\Delta}, \underline{\hat{u}}^{\Delta}, \underline{\hat{v}}^{\Delta}\right) & \leq \lim _{\Delta \rightarrow 0} U^{P \Delta}\left(\mu^{\Delta *}, \underline{\hat{\mu}}^{\Delta}, \underline{\hat{v}}^{\Delta}\right) \\
& =U^{P}\left(\mu^{*}, \underline{\hat{u}}^{\Delta *}, \underline{\hat{v}}^{\Delta *}\right) \leq U^{P *} .
\end{aligned}
$$


From Proposition 3 and (A.27), we also know that there exists a sequence $\left\{\mu^{\Delta}, \underline{u}^{\Delta \prime}, \underline{v}^{\Delta \prime}\right\}$ such that for any $\left.\Delta, \mu^{\Delta}, \underline{u}^{\Delta \prime}, \underline{v}^{\Delta \prime}\right) \in I C(\Delta)$ and moreover $\lim _{\Delta \rightarrow 0} U^{P \Delta}\left(\mu^{\Delta}, \underline{u}^{\Delta \prime}, \underline{v}^{\Delta \prime}\right)=$ $U^{P *}$. Given the definition of $\left\{\mu^{\Delta *}, \hat{u}_{1}^{\Delta *}, \hat{v}_{1}^{\Delta *}, \ldots, \hat{u}_{N}^{\Delta *}, \hat{v}_{N}^{\Delta *}\right\}$ it follows that

$$
\lim \inf _{\Delta \rightarrow 0} U^{P \Delta}\left(\mu^{\Delta *}, \underline{\hat{u}}^{\Delta *}, \underline{\hat{v}}^{\Delta *}\right) \geq U^{P *} .
$$

Upon combining (A.29) and (A.30), we conclude that

$$
\lim _{\Delta \rightarrow 0} U^{P \Delta}\left(\mu^{\Delta *}, \underline{\hat{u}}^{\Delta *}, \underline{\hat{v}}^{\Delta *}\right)=\lim _{\Delta \rightarrow 0} U^{P \Delta}\left(\mu^{\Delta *}, \underline{\hat{u}}^{\Delta}, \underline{\hat{v}}^{\Delta}\right)=U^{P}\left(\mu^{*}, \underline{\hat{u}}^{\Delta *}, \underline{\hat{v}}^{\Delta *}\right)=U^{P *} .
$$

For any $\Delta$, let $\left(\underline{u}^{\Delta \prime \prime}, \underline{v}^{\Delta \prime \prime}\right)$ be an arbitrary convex combination of $\left(\underline{\hat{u}}^{\Delta *}, \underline{\hat{v}}^{\Delta *}\right)$ and $\left(\underline{\hat{u}}^{\Delta}, \underline{\hat{v}}^{\Delta}\right)$. Since $U^{P \Delta}\left(\mu^{\Delta *}, \cdot, \cdot\right)$ is concave and, by the definition of $\left(\underline{\hat{u}}^{\Delta}, \underline{\hat{v}}^{\Delta}\right), U^{P \Delta}\left(\mu^{\Delta *}, \underline{\hat{u}}^{\Delta *}, \underline{\hat{v}}^{\Delta *}\right) \leq$ $U^{P \Delta}\left(\mu^{\Delta *}, \underline{\hat{u}}^{\Delta}, \underline{\hat{v}}^{\Delta}\right)$ for all $\Delta$, we have $U^{P \Delta}\left(\mu^{\Delta *}, \underline{\hat{u}}^{\Delta *}, \underline{\hat{v}}^{\Delta *}\right) \leq U^{P \Delta}\left(\mu^{\Delta *}, \underline{u}^{\Delta \prime \prime}, \underline{v}^{\Delta \prime \prime}\right) \leq U^{P \Delta}\left(\mu^{\Delta *}, \underline{\hat{u}}^{\Delta}, \underline{\hat{v}}^{\Delta}\right)$ for all $\Delta$, so (A.31) implies:

$$
\lim _{\Delta \rightarrow 0} U^{P \Delta}\left(\mu^{\Delta *}, \underline{u}^{\Delta \prime \prime}, \underline{v}^{\Delta \prime \prime}\right)=U^{P}\left(\mu^{*}, \underline{\hat{u}}^{*}, \underline{\hat{v}}^{*}\right)=U^{P *}
$$

Given that $\left(\underline{\hat{u}}^{*}, \underline{\hat{v}}^{*}\right)$ maximizes the strictly concave function $U^{P}\left(\mu^{*}, \cdot, \cdot\right)$ under the constraint (43) - and any limit point of the sequence $\left\{\underline{u}^{\Delta \prime \prime}, \underline{v}^{\Delta \prime \prime}\right\}$ will also satisfy (43) -, it follows that any convergent subsequence of convex combinations of $\left(\underline{\hat{u}}^{\Delta *}, \underline{\hat{v}}^{\Delta *}\right)$ and $\left(\underline{\hat{u}}^{\Delta}, \underline{\hat{v}}^{\Delta}\right)$ must actually converge to $\left(\underline{\hat{u}}^{*}, \underline{\hat{v}}^{*}\right)$. This in turn implies that the sequence $\left\{\underline{\hat{u}}^{\Delta *}, \underline{\hat{v}}^{\Delta *}\right\}$ itself converges to $\left(\underline{\hat{u}}^{*}, \underline{\hat{v}}^{*}\right)$, so $\left(\mu^{*}, \underline{\hat{u}}^{*}, \underline{\hat{v}}^{*}\right)$ is indeed a limit point of the sequence $\left\{\mu^{\Delta *}, \hat{u}_{1}^{\Delta *}, \hat{v}_{1}^{\Delta *}, \ldots, \hat{u}_{N}^{\Delta *}, \hat{v}_{N}^{\Delta *}\right\}$. This completes the proof of Theorem 2. Q.E.D.

Proof of Proposition 4: Consider an incentive scheme $S^{\Delta}(m)$ that implements $\mu^{\Delta}(t)$. If total profits at $t=1$ are given by $z$, the agent reports

$$
m^{*}(z) \in \arg \max _{m \in M(z)} S^{\Delta}(m)
$$

Consider now a mechanism $s^{\Delta}(z)$ which is defined by $s^{\Delta}(z)=S^{\Delta}\left(m^{*}(z)\right)$. If $m^{*}(z)$ is an optimal announcement given $z$ under $S^{\Delta}(m)$, then $z$ must be an optimal announcement under $s^{\Delta}(z)$. Suppose not. If total profits are given by $z$, then the agent cannot report $\hat{z}>z$. If he reports $\hat{z}<z$, his payoff is

$$
s^{\Delta}(\hat{z}) \equiv S^{\Delta}\left(m^{*}(\hat{z})\right) \leq S^{\Delta}\left(m^{*}(z)\right) \equiv s^{\Delta}(z)
$$


since $M(\hat{z}) \subset M(z)$. Hence, the mechanism $s^{\Delta}(z)$ induces the agent to report total profits truthfully. Furthermore, it follows from (A.34) that $s^{\Delta}(z)$ is non-decreasing.

We now have to show that $s^{\Delta}(z)$ induces the agent to choose the same path of action $\mu^{\Delta}(t)$ as $S^{\Delta}(m)$. Since $S^{\Delta}(m)$ implements $\mu^{\Delta}(t)$ it must be the case that

$$
E U^{A}\left(S^{\Delta}\left(m^{*}(z)\right) \mid \mu^{\Delta}(t)\right) \geq E U^{A}\left(S^{\Delta}\left(m^{*}(z)\right) \mid \hat{\mu}^{\Delta}(t)\right)
$$

for all admissible paths of actions $\hat{\mu}^{\Delta}(t)$. However, since $s^{\Delta}(z)$ induces the agent to report total profits truthfully, we have

$$
\begin{aligned}
E U^{A}\left(s^{\Delta}(z) \mid \mu^{\Delta}(t)\right) & =E U^{A}\left(S^{\Delta}\left(m^{*}(z)\right) \mid \mu^{\Delta}(t)\right) \\
& \geq E U^{A}\left(S^{\Delta}\left(m^{*}(z)\right) \mid \hat{\mu}^{\Delta}(t)\right)=E U^{A}\left(s^{\Delta}(z) \mid \hat{\mu}^{\Delta}(t)\right)
\end{aligned}
$$

for all admissible paths of actions $\hat{\mu}^{\Delta}(t)$. Hence, $s^{\Delta}(z)$ also implements $\mu^{\Delta}(t)$.

Finally, since $s^{\Delta}(z)$ implements the same time path of actions and yields the same payments to the agent as the old contract $S^{\Delta}(m)$, the expected utilities of the principal and the agent must also be the same.

Q.E.D.

Proof of Theorem 3: Note first that

$$
Z_{i}^{\Delta}\left(\frac{1}{\Delta}\right)=X_{i}^{\Delta}\left(\frac{1}{\Delta}\right)+\sum_{\tau=1}^{1 / \Delta} \mu_{i}^{\Delta, \tau}
$$

Hence, we can write $s^{\Delta}(\cdot)$ as a function of $x=\sum_{i=1}^{N} X_{i}^{\Delta}\left(\frac{1}{\Delta}\right)$ rather than $z$. Furthermore, if $s(\cdot)$ is nondecreasing in $x$, it is also nondecreasing in $z$.

For any $\Delta$, let $\tilde{\mu}^{\Delta, \tau}, \tau=1, \ldots, \frac{1}{\Delta}$, and $s^{\Delta}(\cdot)$ be a control strategy and an incentive scheme that solve the principal's problem in the discrete-time problem with period length $\Delta$. Formally, the control strategy $\tilde{\mu}^{\Delta, \tau}, \tau=1, \ldots \frac{1}{\Delta}$, and incentive scheme $s^{\Delta}(\cdot)$ maximize the principal's expected payoff,

$$
E\left[\sum_{\tau=1}^{1 / \Delta} \sum_{i=1}^{N} \Delta \tilde{\mu}_{i}^{\Delta, \tau} \Leftrightarrow s^{\Delta}\left(\sum_{i=1}^{N} X_{i}^{\Delta}\left(\frac{1}{\Delta}\right)\right)\right]
$$

subject to the constraints

(IR) If the agent chooses the control path $\tilde{\mu}^{\Delta, \tau}, \tau=1, \ldots, \frac{1}{\Delta}$ he gets at least his reservation utility $\Leftrightarrow e^{-r w}$. 
(IC) Given the incentive scheme $s^{\Delta}(\cdot)$ it is indeed optimal for the agent to choose the control path $\tilde{\mu}^{\Delta, \tau}$.

(MS) The sharing rule $s^{\Delta}(x)$ is nondecreasing in $x$.

By Holmström and Milgrom (1987, Theorem 4) we know that the control strategy $\hat{\mu}^{\Delta, \tau}$ is implemented by sharing rule $s^{\Delta}$ if and only if cumulative payments under this scheme can be written as a sum of payments under incentive schemes $s^{\Delta, \tau}$ that would implement $\hat{\mu}^{\Delta, \tau}$ in the static problem, $\tau=1, \ldots, \frac{1}{\Delta}$. Furthermore, we have shown in Proposition 2 of Section 4 that the action $\tilde{\mu}^{\Delta, \tau}$ can be implemented by $\tilde{s}^{\Delta, \tau}$ only if $\tilde{s}^{\Delta, \tau}$ satisfies

$$
\begin{aligned}
\tilde{s}_{i}^{\Delta, \tau}=\Delta \hat{c}\left(\mu^{\Delta}\right) \Leftrightarrow \frac{1}{r} \ln \left[1 \Leftrightarrow r\left(\hat{c}_{i}\left(\mu^{\Delta, \tau}\right) \Leftrightarrow u_{i}^{\Delta *, \tau}+v_{i}^{\Delta *, \tau}\right) k_{i} \Delta^{\frac{1}{2}}\right. \\
\left.+r \sum_{j=0}^{N} p_{j}^{\Delta}\left(\hat{c}_{j}\left(\mu^{\Delta, \tau}\right) \Leftrightarrow u_{j}^{\Delta *, \tau}+v_{j}^{\Delta *, \tau}\right) k_{j} \Delta^{\frac{1}{2}}\right]
\end{aligned}
$$

for some vectors $\underline{\hat{u}}^{\Delta, \tau}=\left(\hat{u}_{1}^{\Delta, \tau}, \ldots, \hat{u}_{N}^{\Delta, \tau}\right), \underline{\hat{v}}^{\Delta, \tau}=\left(\hat{v}_{1}^{\Delta, \tau}, \ldots, \hat{v}_{N}^{\Delta, \tau}\right)$. It follows that the principal's maximal expected payoff in problem (A.38) with the constraints (IR), (IC), and (MS) is no larger than the payoff he would obtain if he chose a control strategy $\hat{\mu}^{\Delta, \tau}$, an incentive scheme $\hat{s}^{\Delta}$, and vectors $\underline{\hat{u}}^{\Delta, \tau}, \underline{\hat{v}}^{\Delta, \tau}$ to maximize the expression:

$$
\begin{gathered}
E\left[\sum_{\tau=1}^{1 / \Delta} \sum_{i=1}^{N} \Delta \mu_{i}^{\Delta, \tau} \sum_{\tau=1}^{1 / \Delta} \Delta \hat{c}\left(\mu^{\Delta, \tau}\right)+\frac{1}{r} \sum_{\tau=1}^{1 / \Delta} \sum_{i=1}^{N}\left(\frac{\tilde{X}_{i}^{\Delta, \tau}}{k_{i} \Delta^{\frac{1}{2}}}+p_{i}^{\Delta}\right)\right. \\
\left.\cdot \ln \left(1 \Leftrightarrow r\left(\hat{c}_{i}\left(\mu^{\Delta, \tau}\right) \Leftrightarrow u_{i}^{\Delta *, \tau}+v_{i}^{\Delta *, \tau}\right) k_{i} \Delta^{\frac{1}{2}}+r \sum_{j=0}^{N} p_{j}^{\Delta}\left(\hat{c}_{j}\left(\mu^{\Delta, \tau}\right) \Leftrightarrow u_{j}^{\Delta *, \tau}+v_{j}^{\Delta *, \tau}\right) k_{j} \Delta^{\frac{1}{2}}\right) \Leftrightarrow w\right]
\end{gathered}
$$

under the constraints that

$$
\begin{aligned}
& s^{\Delta}\left(\sum_{i=1}^{N} \tilde{X}_{i}^{\Delta}\left(\frac{1}{\Delta}\right)\right)=w+\sum_{\tau=1}^{1 / \Delta} \Delta \hat{c}\left(\mu_{i}^{\Delta, \tau}\right) \Leftrightarrow \frac{1}{r} \sum_{\tau=1}^{1 / \Delta} \sum_{i=1}^{N}\left(\frac{\tilde{X}_{i}^{\Delta, \tau}}{k_{i} \Delta^{\frac{1}{2}}}+p_{i}^{\Delta}\right) \\
& \cdot \ln \left(1 \Leftrightarrow r\left(\hat{c}_{i}\left(\mu^{\Delta, \tau}\right) \Leftrightarrow u_{i}^{\Delta *, \tau}+v_{i}^{\Delta *, \tau}\right) k_{i} \Delta^{\frac{1}{2}}+r \sum_{j=0}^{N} p_{j}^{\Delta}\left(\hat{c}_{j}\left(\mu^{\Delta, \tau}\right) \Leftrightarrow u_{j}^{\Delta *, \tau}+v_{j}^{\Delta *, \tau}\right) k_{j} \Delta^{\frac{1}{2}}\right)
\end{aligned}
$$

with probability one, and (MS) $s^{\Delta}(\cdot)$ is a nondecreasing function.

Let $\hat{\mu}^{\Delta, \tau}, \hat{s}^{\Delta, \tau}, \underline{\hat{u}}^{\Delta, \tau}, \underline{\hat{v}}^{\Delta, \tau}$ be a solution to this problem. By Proposition $2, \underline{\hat{u}}^{\Delta, \tau}$ and $\underline{\hat{v}}^{\Delta, \tau}$ belong to the compact set $[0, G]^{N}$ where $G$ is the $\operatorname{maximum}$ of $\max _{i} \frac{\gamma(\mu)}{k_{i}}$ over $K$. Using a Taylor expansion for the logarithmic term, we therefore find that for any $\eta>0$ 
the value of (A.38) is no larger than

$E\left[\sum_{\tau=1}^{1 / \Delta} \sum_{i=1}^{N} \Delta \hat{\mu}_{i}^{\Delta, \tau} \Leftrightarrow \sum_{i=1}^{N} \Delta \hat{c}\left(\hat{\mu}^{\Delta, \tau}\right) \Leftrightarrow \frac{r}{2} \sum_{\tau=1}^{1 / \Delta} \sum_{i=1}^{N} \Delta \hat{p}_{i}\left(\gamma_{i}\left(\hat{\mu}^{\Delta, \tau}\right) k_{i} \Leftrightarrow \sum_{i=1}^{N} \hat{p}_{j} \gamma_{j}\left(\hat{\mu}^{\Delta, \tau}\right) k_{j}\right)^{2}\right] \Leftrightarrow w \Leftrightarrow \eta$,

where $\gamma_{i}\left(\mu^{\Delta, \tau}\right)=\hat{c}_{i}\left(\mu^{\Delta, \tau}\right) \Leftrightarrow u_{i}^{\Delta *, \tau}+v_{i}^{\Delta *, \tau}$, if $\Delta$ is sufficiently close to zero. Here we have made use of the fact that the expected value of the linear term of the Taylor expansion is zero and the fact that for each $\tau$ and $i$, conditional on $\hat{\mu}^{\Delta, \tau}$, the coefficient $\left(\tilde{X}_{i}^{\Delta, \tau} / k_{i} \Delta^{\frac{1}{2}}+\right.$ $\left.p_{i}^{\Delta}\left(\hat{\mu}^{\Delta, \tau}\right)\right)=\tilde{A}_{i}^{\Delta, \tau}$ of the logarithmic term has expected value $p_{i}^{\Delta}\left(\hat{\mu}^{\Delta, \tau}\right)=\hat{p}_{i}+O\left(\Delta^{\frac{1}{2}}\right)$ and variance $p_{i}^{\Delta}\left(\hat{\mu}^{\Delta, \tau}\right)\left(1 \Leftrightarrow p_{i}^{\Delta}\left(\hat{\mu}^{\Delta, \tau}\right)\right)$, so for the quadratic term of the Taylor expansion, which is proportional to $\Delta$, this coefficient may be approximated by $\hat{p}_{i}$ if $\Delta$ is sufficiently small.

At this point it is convenient to rewrite (A.42) and (A.38) using continuous-time notation. In continuous-time notation, our preceding argument can be summarized as saying that for any $\eta>0$ and any sufficiently small $\Delta$, in the $\frac{1}{\Delta}$-period problem, the principal's maximal expected payoff is no larger than $\eta$ plus the maximum of the expression

$E\left[\int_{0}^{1} \sum_{i=1}^{N} \hat{\mu}_{i}^{\Delta}(t) d t \Leftrightarrow \int_{0}^{1} \hat{c}\left(\hat{\mu}^{\Delta}(t)\right) d t \Leftrightarrow \frac{r}{2} \int_{0}^{1} \sum_{i=1}^{N} \hat{p}_{i}\left(\gamma_{i}\left(\hat{\mu}^{\Delta}(t)\right) k_{i} \Leftrightarrow \sum_{j=1}^{N} \hat{p}_{j} \gamma_{j}\left(\hat{\mu}^{\Delta}(t)\right) k_{j}\right)^{2} d t\right] \Leftrightarrow w$

with respect to the control strategy $\mu^{\Delta}(\cdot)$ and an incentive scheme $s^{\Delta}(\cdot)$ satisfying

(PL) $\mu^{\Delta}(\cdot)$ is piecewise linear with $\mu^{\Delta}(t)=\mu^{\Delta,[t / \Delta]}$, and

$$
\begin{aligned}
& s^{\Delta}\left(\sum_{i=1}^{N} X_{i}^{\Delta}(1)\right)=w+\int_{0}^{1} \hat{c}\left(\mu^{\Delta}(t)\right) d t \\
& \quad \Leftrightarrow \frac{1}{r} \int_{0}^{1} \sum_{i=1}^{N} \frac{1}{k_{i} \Delta^{\frac{1}{2}}} \ln \left(1 \Leftrightarrow r \gamma_{i}\left(\mu^{\Delta}(t)\right) k_{i} \Delta^{\frac{1}{2}}+r \sum_{j=0}^{N} p_{j}^{\Delta} \gamma_{j}\left(\mu^{\Delta}(t)\right) k_{j} \Delta^{\frac{1}{2}}\right) d X_{i}^{\Delta}(t) \\
& \quad \Leftrightarrow \frac{1}{r} \int_{0}^{1} \sum_{i=1}^{N} p_{i}^{\Delta}\left(\mu^{\Delta}(t)\right) \ln \left(1 \Leftrightarrow r \gamma_{i}\left(\mu^{\Delta}(t)\right) k_{i} \Delta^{\frac{1}{2}}+r \sum_{j=0}^{N} p_{j}^{\Delta} \gamma_{j}\left(\mu^{\Delta}(t)\right) k_{j} \Delta^{\frac{1}{2}}\right) d t
\end{aligned}
$$

with probability one, and (MS) $s^{\Delta}(\cdot)$ is a nondecreasing function.

By Proposition B.1 in Appendix B we know that as $\Delta$ goes to zero, the disturbance process converges uniformly to a Brownian motion with initial value $B(0)=0$, zero drift and covariance matrix $\boldsymbol{\Sigma}$. Given that the range of this Brownian motion can be identified 
with the set of all continuous functions of $[0,1]$ into $I R^{N}$, it follows that as $\Delta$ goes to zero the set of pairs of control strategies $\mu^{\Delta}(\cdot)$ and incentive schemes $s^{\Delta}(\cdot)$ satisfying (A.44) as well as (MS) shrinks to the set of pairs of control strategies $\mu(\cdot)$ and incentive schemes $s(\cdot)$ satisfying

$$
\begin{aligned}
& s^{\Delta}\left(\sum_{i=1}^{N} X_{i}^{\Delta}(1)\right)=w+\int_{0}^{1} \hat{c}\left(\mu^{\Delta}(t)\right) d t \\
& \quad+\int_{0}^{1} \sum_{i=1}^{N} \gamma_{i}(\mu(t)) d X_{i}(t) d t+\frac{r}{2} \int_{0}^{1} \gamma^{\prime}(\mu(t)) \Sigma\left[\gamma^{\prime}(\mu(t))\right]^{T} d t
\end{aligned}
$$

with probability one and (MS) $s(\cdot)$ is a nondecreasing function. Hence, for all $\eta>0$ and any sufficiently small $\Delta$, the maximum value of (A.43) under the constraints (PL), (A.44) and (MS) is no less than $\eta$ plus the maximum value of

$$
\int_{0}^{1} \sum_{i=1}^{N} \mu_{i}(t) d t \Leftrightarrow \int_{0}^{1} \hat{c}(\mu(t)) d t \Leftrightarrow \frac{r}{2} \int_{0}^{1} \gamma^{\prime}(\mu(t)) \Sigma\left[\gamma^{\prime}(\mu(t))\right]^{T} d t \Leftrightarrow w
$$

under the constraints (A.45) and (MS).

Now compare the problem of maximizing (A.46) under the constraints (A.45) and (MS) to the continuous-time problem with controls $\mu_{i}(t)$ as discussed in Appendix C. In this appendix, as well as in Holmstöm and Milgrom (1987, Theorem 8), the principal maximizes the same payoff function but the incentive scheme can be a function of the entire time path of the aggregate $\sum_{i=1}^{N} X_{i}(\cdot)$. Proposition C.2 shows that this problem is solved by a constant control path $\mu^{*}$ and a linear sharing rule $s^{*}(z)=\alpha_{0}+\alpha_{1} z$ that depends only on accumulated total profits at time 1 , z, with $\alpha_{1}=\gamma_{1}\left(\mu^{*}\right)=\gamma_{2}\left(\mu^{*}\right)=\ldots=\gamma_{N}\left(\mu^{*}\right)>0$ and $\alpha_{0}=\hat{c}\left(\mu^{*}\right) \Leftrightarrow \hat{c}^{\prime}\left(\mu^{*}\right)^{T} \mu^{*}+\frac{r}{2} \gamma^{\prime}\left(\mu^{*}\right) \Sigma\left[\gamma^{\prime}\left(\mu^{*}\right)\right]^{T}$. Clearly, a constant time path of actions satisfies our restriction (PL). Furthermore, the incentive scheme also satisfies (MS') and (IC). Hence, the optimal solution to Holmström and Milgrom's optimization problem is also the solution to the problem of maximizing (A.46) under the constraints (A.45) and (MS).

If we substitute the maximizer $\mu^{*}$ on the right-hand side of (A.46), we find that for any $\eta>0$ and any sufficiently small $\Delta$, the maximum value of the principal's expected payoff (A.38) in the $\frac{1}{\Delta}$-period discrete-time problem with the constraints (IR), (IC) and (MS) is no larger than

$$
\sum_{i=1}^{N} \mu_{i}^{*} \Leftrightarrow \hat{c}\left(\mu^{*}\right) \Leftrightarrow \frac{r}{2} \gamma^{\prime}\left(\mu^{*}\right) \Sigma\left[\gamma^{\prime}\left(\mu^{*}\right)\right]^{T} \Leftrightarrow w+2 \eta
$$


Using the entire approximation argument in reverse, we also find that for given $\eta>0$ and any sufficiently small $\Delta,(\mathrm{A} .47)$ is no larger than

$$
E\left[\sum_{\tau=1}^{1 / \Delta} \sum_{i=1}^{N} \mu_{i}^{\Delta *} \Leftrightarrow s\left(\sum_{i=1}^{N} \tilde{X}_{i}^{\Delta}\left(\frac{1}{\Delta}\right)\right)\right]+3 \eta,
$$

where for any $\Delta, \mu^{\Delta *}$ is the agent's (unique) optimal action when faced with the linear scheme $s(\cdot)$. Upon setting $\eta=\epsilon / 3$, we obtain the conclusion of Theorem 3. Q.E.D.

\section{Appendix B}

In this appendix we discuss the relationship between discrete-time and continous-time strategies and outcome processes when the agent's choice at any one time may depend on the history of the process up to this time. In the multi-period discrete-time model with period length $\Delta$, the agent chooses a sequence of possibly history-dependent controls $\left\{\tilde{\mu}^{\Delta, \tau}\right\}_{\tau=1}^{1 / \Delta}$. This choice generates a sequence $\left\{\tilde{\pi}^{\Delta, \tau}\right\}_{\tau=1}^{1 / \Delta}$ of random profit levels. We assume that for each $\tau$, the agent's control choice $\tilde{\mu}^{\Delta, \tau}$ is given by a - possibly degenerate - function of profit realizations prior to $\tau$, i.e., that we can write

$$
\tilde{\mu}^{\Delta, \tau}=\hat{\mu}^{\Delta, \tau}\left(\tilde{\pi}^{\Delta, 1}, \ldots, \tilde{\pi}^{\Delta, \tau-1}\right)
$$

for all $\tau$, and we identify the agent's strategy with the sequence of functions $\left\{\hat{\mu}^{\Delta, \tau}\right\}_{\tau=1}^{1 / \Delta}$. The range of each function $\hat{\mu}^{\Delta, \tau}$ is taken to be the compact set $K=\prod_{i=1}^{N}\left[\underline{m}_{i}, M_{i}\right]$. Given the control strategy $\left\{\hat{\mu}^{\Delta, \tau}\right\}_{\tau=1}^{1 / \Delta}$ the profit sequence $\left\{\tilde{\pi}^{\Delta, \tau}\right\}_{\tau=1}^{1 / \Delta}$ is assumed to satisfy the condition that for each $\tau \in\{1, \ldots, 1 / \Delta\}, \tilde{\pi}^{\Delta, \tau}$ takes values in $\left\{\pi_{0} \Delta^{\frac{1}{2}}, \ldots, \pi_{N} \Delta^{\frac{1}{2}}\right\}$ and, for given $\pi^{\Delta, 1}, \ldots, \pi^{\Delta, \tau-1}$

$$
\begin{gathered}
\operatorname{Prob}\left(\left\{\tilde{\pi}^{\Delta, \tau}=\pi_{i} \Delta^{\frac{1}{2}}\right\} \quad \mid \quad \tilde{\pi}^{\Delta, 1}=\pi^{\Delta, 1}, \ldots, \tilde{\pi}^{\Delta, \tau-1}=\pi^{\Delta, \tau-1}\right) \\
=p_{i}^{\Delta}\left(\hat{\mu}^{\Delta, \tau}\left(\pi^{\Delta, 1}, \ldots, \pi^{\Delta, \tau-1}\right)\right)
\end{gathered}
$$

As in the text, a given control strategy $\left\{\hat{\mu}^{\Delta, \tau}\right\}_{\tau=1}^{1 / \Delta}$ and associated profit sequence $\left\{\tilde{\pi}^{\Delta, \tau}\right\}_{\tau=1}^{1 / \Delta}$ are used to define counting variables $\tilde{A}_{i}^{\Delta, \tau}, i=0,1, \ldots, N, \tau=1, \ldots, \frac{1}{\Delta}, \Delta=1, \frac{1}{2}, \ldots$ such that $\tilde{A}_{i}^{\Delta, \tau}=1 \Leftrightarrow \tilde{\pi}^{\Delta, \tau}=\pi_{i} \Delta^{\frac{1}{2}}$ and $\tilde{A}_{i}^{\Delta, \tau}=0 \Leftrightarrow \tilde{\pi}^{\Delta, \tau} \neq \pi_{i} \Delta^{\frac{1}{2}}$. From (B.2) one obviously has:

$$
E\left[\tilde{A}_{i}^{\Delta, \tau} \mid \tilde{\pi}^{\Delta, 1}=\pi^{\Delta, 1}, \ldots, \tilde{\pi}^{\Delta, \tau-1}=\pi^{\Delta, \tau-1}\right] \equiv p_{i}^{\Delta}\left(\hat{\mu}^{\Delta, \tau}\left(\pi^{\Delta, 1}, \ldots, \pi^{\Delta, \tau-1}\right)\right)
$$


It follows that if we define

$$
\tilde{X}_{i}^{\Delta, \tau}=k_{i} \Delta^{\frac{1}{2}}\left(\tilde{A}_{i}^{\Delta, \tau} \Leftrightarrow p_{i}^{\Delta}\left(\hat{\mu}^{\Delta, \tau}\left(\pi^{\Delta, 1}, \ldots, \pi^{\Delta, \tau-1}\right)\right)\right.
$$

we have

$$
E\left[\tilde{X}_{i}^{\Delta, \tau} \mid \tilde{\pi}^{\Delta, 1}=\pi^{\Delta, 1}, \ldots, \tilde{\pi}^{\Delta, \tau-1}=\pi^{\Delta, \tau-1}\right] \equiv 0
$$

i.e., for any $\Delta$ the process $\left\{\left(\tilde{X}_{1}^{\Delta}(\tau), \ldots, \tilde{X}_{N}^{\Delta}(\tau)\right)\right\}_{\tau=1}^{1 / \Delta}$ is a martingale. Again using a linear interpolation to embed the processes $\left\{\left(\tilde{X}_{1}^{\Delta}(\tau), \ldots, \tilde{X}_{N}^{\Delta}(\tau)\right)\right\}_{\tau=1}^{1 / \Delta}$ in a continuous-time formulation, we write for any $i$ and any $t \in[0,1]$ :

$$
X_{i}^{\Delta}(t)=\left(1 \Leftrightarrow \frac{t}{\Delta}+\left[\frac{t}{\Delta}\right]\right) \tilde{X}_{i}^{\Delta}\left(\left[\frac{t}{\Delta}\right]\right)+\left(\frac{t}{\Delta} \Leftrightarrow\left[\frac{t}{\Delta}\right]\right) \tilde{X}_{i}^{\Delta}\left(\left[\frac{t}{\Delta}\right]+1\right)
$$

where again $\left[\frac{t}{\Delta}\right]$ denotes the greatest integer less than or equal to $\frac{t}{\Delta}$. For any $\Delta$, $\left(X_{1}^{\Delta}(\cdot), \ldots, X_{N}^{\Delta}(\cdot)\right)$ is a random function taking values in the space $C^{N}[0,1]$ of continuous functions from $[0,1]$ into $\mathbb{R}^{N}$. The distribution of $\left(X_{1}^{\Delta}(\cdot), \ldots, X_{N}^{\Delta}(\cdot)\right)$ depends on the chosen control strategy of the agent.

Proposition B.1 For $\Delta=1, \frac{1}{2}, \frac{1}{3}, \ldots$, let $\left\{\hat{\mu}^{\Delta, \tau}\right\}_{\tau=1}^{1 / \Delta}$ be a control strategy taking values in the compact set $K=\prod_{i=1}^{N}\left[\underline{m}_{i}, M_{i}\right]$, and consider the induced process $\left(X_{1}^{\Delta}(\cdot), \ldots, X_{N}^{\Delta}(\cdot)\right)$. As $\Delta$ converges to zero, the processes $\left(X_{1}^{\Delta}(\cdot), \ldots, X_{N}^{\Delta}(\cdot)\right)$ converge in distribution to the Gaussian process $B(\cdot)$ with initial value $B(0)=$ 0 , zero drift, and covariance matrix $\boldsymbol{\Sigma}$.

Proof: For any $\Delta$, let $\left\{\tilde{\pi}^{\Delta, \tau}\right\}_{\tau=1}^{1 / \Delta}$ be the random profit sequence that is induced by the control strategy $\left\{\hat{\mu}^{\Delta, \tau}\right\}_{\tau=1}^{1 / \Delta}$. For any $\Delta$ and any $t \in[0,1]$, let $\mathcal{F}_{t}^{\Delta}$ be the $\sigma$-algebra on the underlying probability space that is generated by the random variables $\tilde{\pi}^{\Delta, 1}, \ldots, \tilde{\pi}^{\Delta,[t / \Delta]}$. Fix some vector $q \in I R^{N}$, and consider the stochastic process $X_{q}^{\Delta}(\cdot)$ on $[0,1]$ such that for any $t \in[0,1]$,

$$
X_{q}^{\Delta}(t)=\sum_{i=1}^{N} q_{i} X_{i}^{\Delta}(t)
$$

For any $t$ and $\Delta,($ B.5) and (B.6) yield:

$$
E\left[X_{q}^{\Delta}([t / \Delta] \Delta+\Delta) \Leftrightarrow X_{q}^{\Delta}([t / \Delta] \Delta) \mid \mathcal{F}_{t}^{\Delta}\right]=E\left[\sum_{i=1}^{N} q_{i} \tilde{X}_{i}^{\Delta,[t / \Delta]+\Delta} \mid \mathcal{F}_{t}^{\Delta}\right]=0
$$


For any two vectors $q^{1}, q^{2} \in \mathbb{R}^{N}$, one also has:

$$
\begin{aligned}
E\left[\left(X_{q^{1}}^{\Delta}([t / \Delta] \Delta+\Delta) \Leftrightarrow\right.\right. & X_{q^{1}}^{\Delta}([t / \Delta] \Delta)\left(X_{q^{2}}^{\Delta}([t / \Delta] \Delta+\Delta) \Leftrightarrow X_{q^{2}}^{\Delta}([t / \Delta] \Delta) \mid \mathcal{F}_{t}^{\Delta}\right] \\
= & E\left[\sum_{i=1}^{N} \sum_{j=1}^{N} q_{i}^{1} \tilde{X}_{i}^{\Delta,[t / \Delta]+\Delta} q_{j}^{2} \tilde{X}_{j}^{\Delta,[t / \Delta]+\Delta} \mid \mathcal{F}_{t}^{\Delta}\right] \\
= & E\left[\sum_{i=1}^{N} q_{i}^{1} q_{i}^{2} k_{i}^{2} \Delta\left(1 \Leftrightarrow p_{i}^{\Delta}\left(\tilde{\mu}^{\Delta,[t / \Delta]}\right)\right) p_{i}^{\Delta}\left(\tilde{\mu}^{\Delta,[t / \Delta]}\right) \mid \mathcal{F}_{t}^{\Delta}\right] \\
& \Leftrightarrow E\left[\sum_{i=1}^{N} \sum_{j \neq i} q_{i}^{1} q_{j}^{2} k_{i} k_{j} \Delta p_{i}^{\Delta}\left(\tilde{\mu}^{\Delta,[t / \Delta]}\right) p_{j}^{\Delta}\left(\tilde{\mu}^{\Delta,[t / \Delta]}\right) \mid \mathcal{F}_{t}^{\Delta}\right] \\
= & \sum_{i=1}^{N} q_{i}^{1} q_{i}^{2} k_{i}^{2} \Delta \hat{p}_{i} \Leftrightarrow \sum_{i=1}^{N} \sum_{j=1}^{N} q_{i}^{1} q_{j}^{2} k_{i} k_{j} \Delta \hat{p}_{i} \hat{p}_{j}+\Delta O(\Delta) \\
= & \sum_{i=1}^{N} \sum_{j=1}^{N} q_{i}^{1} q_{j}^{2} \sigma_{i j} \Delta+\Delta O(\Delta)
\end{aligned}
$$

where $\sigma_{i j}$ is the ij-th element of the matrix $\Sigma$ (see (23) in the text) and $O(\Delta)$ is a term that goes to zero when $\Delta$ goes to zero, uniformly in strategies and histories.

Now consider the vector process

$$
X_{Q}^{\Delta}=\left(X_{q^{1}}^{\Delta}, \ldots, X_{q^{N}}^{\Delta}\right)
$$

where $q^{1}, \ldots, q^{N}$ are eigenvectors of the matrix $\Sigma$, normed so that $q^{r \prime} q^{r}=\lambda_{r}^{-1}$ where $\lambda_{r}$ is the eigenvalue of $\boldsymbol{\Sigma}$ that corresponds to $q^{r}$. Given that $\boldsymbol{\Sigma}$ is a symmetric, positive definite matrix, the eigenvectors $q^{r}, q^{s}, r \neq s$, are mutually orthogonal. For this choice of $q^{1}, \ldots, q^{N},(\mathrm{~B} .9)$ yields:

$$
\begin{aligned}
E\left[\left(X_{q^{r}}^{\Delta}([t / \Delta] \Delta+\Delta) \Leftrightarrow\right.\right. & X_{q^{r}}^{\Delta}([t / \Delta] \Delta)\left(X_{q^{s}}^{\Delta}([t / \Delta] \Delta+\Delta) \Leftrightarrow X_{q^{s}}^{\Delta}([t / \Delta] \Delta) \mid \mathcal{F}_{t}^{\Delta}\right] \\
& =\sum_{i=1}^{N} \sum_{j=1}^{N} q_{i}^{r} q_{j}^{s} \sigma_{i j} \Delta+\Delta O(\Delta) \\
& =\sum_{i=1}^{N} q_{i}^{r} \lambda_{s} q_{i}^{s} \Delta+\Delta O(\Delta) \\
& =\delta_{r s} \Delta+\Delta O(\Delta)
\end{aligned}
$$

where $\delta_{i j}=1$ if $i=j$ and $\delta_{i j}=0$ if $i \neq j$.

Finally we also have, for any $\varepsilon>0$, any $\mathrm{t}$ and any i,

$$
\operatorname{Prob}\left\{\mid X_{q^{i}}^{\Delta}([t / \Delta] \Delta+\Delta) \Leftrightarrow X_{q^{i}}^{\Delta}\left([t / \Delta] \Delta|\geq \varepsilon| \mathcal{F}_{t}^{\Delta}\right\}=0\right.
$$


for any $\Delta<\left[\varepsilon / \max \left|k_{j}\right|\right]^{2}$.

From (B.8), (B.10), and (B.11), one easily sees that the processes $X_{Q}^{\Delta}, \Delta=1, \frac{1}{2}, \frac{1}{2}, \ldots$ satisfy the assumptions of Theorems 6 and 7 of Gihman and Skorohod (1979, p.195). It follows that for any $t$, as $\Delta$ converges to zero, the conditional distributions of $\left\{X_{Q}^{\Delta}\left(t^{\prime}\right)\right\}_{t^{\prime} \in[t, 1]}$ given $\mathcal{F}_{t}^{\Delta}$ converge to the conditional distribution of a standard $N$-dimensional Brownian motion $W($.$) on [t, 1]$ given the "initial" value $W(t)$. In particular the overall distributions of the processes $X_{Q}^{\Delta}$ on $[0,1]$ converge to the distribution of a standard Browninan motion on $[0,1]$ with ininital value $W(0)=0$.

Given this convergence result, the proposition follows from Theorem 5.1 of Billingsley (1968, p.30) in conjunction with the observation that for any $\Delta$, one has $\left(X_{1}^{\Delta}(),. \ldots, X_{N}^{\Delta}().\right)=$ $\left(Q^{\prime}\right)^{-1} X_{Q}^{\Delta}$.

Q.E.D.

\section{Appendix $\mathrm{C}$}

In this appendix we analyse the continuous-time agency problem with controls restricted to a product of compact intervals. In the continuous-time model, the principal's problem is to choose a functional $s(\cdot)$ on the space of continuous functions from $[0,1]$ into $\mathbb{R}^{N}$ and an admissible control process $\mu(\cdot)$, taking values in $\prod_{i=1}^{N}\left[\underline{m}_{i}, M_{i}\right]$, so as to maximize the expectation of $\int_{0}^{1} \sum_{i=1}^{N} \mu_{i}(t) d t \Leftrightarrow s(X)$ subject to the constraint $(i)$ that given the incentive scheme $s(\cdot)$, the agent is willing to choose the control process $\mu(\cdot)$, and $(i i)$ that the resulting expected utility of the agent be at least as large as $\Leftrightarrow \exp (\Leftrightarrow r w)$, his expected utility elsewhere. If an incentive scheme $s(\cdot)$ and a control process $\mu(\cdot)$ satisfy both these constraints, we say that $s(\cdot)$ implements $\mu(\cdot)$. For a precise definition of admissibility of a control process, as well as other details of the formulation of the principal's problem and its analysis, we refer the reader to Schättler and Sung (1993). Under our assumptions, their results are easily adapted to yield:

Proposition C.1 An admissible control process $\mu^{*}(\cdot)$ is implementable by some incentive scheme $s^{*}(\cdot)$ if and only if there exist nonnegative-valued adapted processes $u_{i}(\cdot), v_{i}(\cdot), i=1, \ldots, N$, such that for (almost) every $t \in[0,1]$, with probability one, the (possibly history dependent) control $\mu^{*}(t)$ minimizes the 
expresssion

$$
\hat{c}(\mu(t)) \Leftrightarrow \sum_{i=1}^{N}\left[\hat{c}_{i}(\mu(t)) \Leftrightarrow u_{i}(t)+v_{i}(t)\right] \mu_{i}(t)
$$

under the constraints $(i) \mu(t) \in \prod_{i=1}^{N}\left[\underline{m}_{i}, M_{i}\right]$, and $(i i)$ the incentive scheme $s^{*}(\cdot)$ has the representation:

$$
\begin{aligned}
s^{*}(X) & =w+\int_{0}^{1} \hat{c}\left(\mu^{*}(t)\right) d t+\int_{0}^{1} \sum_{i=1}^{N}\left[\hat{c}_{i}\left(\mu^{*}(t)\right) \Leftrightarrow u_{i}(t)+v_{i}(t)\right] d X(t) \\
& +\frac{r}{2} \int_{0}^{1} \sum_{i=1}^{N} \sum_{j=1}^{N}\left[\hat{c}_{i}\left(\mu^{*}(t)\right) \Leftrightarrow u_{i}(t)+v_{i}(t)\right] \sigma_{i j}\left[\hat{c}_{j}\left(\mu^{*}(t)\right) \Leftrightarrow u_{j}(t)+v_{j}(t)\right] d t
\end{aligned}
$$

where $\boldsymbol{\Sigma}=\left(\sigma_{i j}\right)$ is the covariance matrix of the $N$-dimensional Brownian motion that is specified in Theorem 1.

Remark C.1: Given the assumption that $\hat{c}(\cdot)$ is convex, the condition that $\mu^{*}$ minimize (C.1) over the set $K=\prod_{i=1}^{N}\left[\underline{m}_{i}, M_{i}\right]$ is equivalent to the complementary slackness condition that

$$
u_{i}(t)\left[\mu_{i}^{*}(t) \Leftrightarrow \underline{m}_{i}\right]=v_{i}(t)\left[M_{i} \Leftrightarrow \mu_{i}^{*}(t)\right]
$$

for all $i$.

Proof of Proposition C.1: The argument involves a straightforward modification of the proof of Theorem 4.2 of Schättler and Sung (1993, pp. 352f) to take account of the possibility of boundary values of the controls. If $s^{*}(\cdot)$ implements the control process $\mu^{*}(\cdot)$, then by Theorem 4.1, p. 348, of Schättler and Sung, there exist adapted processes $V(\cdot)$ and $\nabla V(\cdot)$, taking values in $I R_{-}$and in $I R_{+}^{N}$ such that for almost every $t$, with probability one, $\mu^{*}(t)$ maximizes $\sum_{i=1}^{N} \nabla V_{i}(t) \mu_{i}(t)+r V(t) \hat{c}(\mu(t))$ over the set $\prod_{i=1}^{N}\left[\underline{m}_{i}, M_{i}\right] .{ }^{18}$ The first-order condition for this problem implies the existence of nonnegative $u_{i}(t), v_{i}(t)$ such that for any $i$,

$$
\frac{\nabla V_{i}(t)}{r V(t)}=\Leftrightarrow\left[\hat{c}_{i}\left(\mu^{*}(t)\right) \Leftrightarrow u_{i}(t)+v_{i}(t)\right]
$$

with $u_{i}(t)\left(\mu_{i}(t) \Leftrightarrow \underline{m}_{i}\right)=v_{i}(t)\left(M_{i} \Leftrightarrow \mu_{i}(t)\right)=0$. For this specification of $u_{i}(t)$ and $v_{i}(t)$, it is then easy to see that $\mu^{*}(t)$ indeed minimizes the expresssion (C.1) over the set $\prod_{i=1}^{N}\left[\underline{m}_{i}, M_{i}\right]$. The representation (C.2) for the incentive scheme $s^{*}(\cdot)$ is obtained by using (C.4) to substitute in the incentive scheme representation given in Theorem 4.1 of Schättler and Sung (1993).

\footnotetext{
${ }^{18}$ The symbol $\nabla$ is only natational and does not stand for a derivative.
} 
Conversely if there exist adapted processes $u_{i}(\cdot), v_{i}(\cdot)$ such that for (almost) every $t$, with probability one, $\mu^{*}(t)$ minimizes the expresssion (C.1) over the set $\prod_{i=1}^{N}\left[\underline{m}_{i}, M_{i}\right]$, then formula (C.2) defines an incentive scheme $s^{*}(\cdot)$, and the argument given in the proof of Theorem 4.2 of Schättler and Sung shows that this incentive scheme implements the control process $\mu^{*}(\cdot)$.

Q.E.D.

Proposition C.1 has the following immediate corollary:

Corollary C.1 An admissible control process $\mu^{*}(\cdot)$ is implementable by an incentive scheme $s^{*}(\cdot)$ taking the form $s^{*}(X(\cdot))=s^{* *}\left(\sum_{i=1}^{N} X_{i}(\cdot)\right)$ if and only if there exist nonnegative-valued adapted processes $u_{i}(\cdot), v_{i}(\cdot), i=1, \ldots, N$, such that for (almost) every $t \in[0,1]$, with probability one, the (possibly history dependent) control $\mu^{*}(t)$ minimizes (C.1) over the set $\prod_{i=1}^{N}\left[\underline{m}_{i}, M_{i}\right]$ and moreover $\hat{c}_{i}\left(\mu^{*}(t)\right) \Leftrightarrow u_{i}(t)+v_{i}(t)$ is the same for all $i$.

Lemma C.1 For any $E \in\left[\sum_{i=1}^{N} \underline{m}_{i}, \sum_{i=1}^{N} M_{i}\right]$, let $\gamma(E)$ be the minimum of $\hat{c}(\mu)$ over the set $\prod_{i=1}^{N}\left[\underline{m}_{i}, M_{i}\right]$ under the constraint that $\sum_{i=1}^{N} \mu_{i}=E$, and let $\mu(E)$ be the corresponding minimizer. The control process $\mu^{*}(\cdot)$ satisfying $\mu^{*}(t)=\mu(E)$ for all $t$ is implementable by an incentive scheme $s^{*}(\cdot)$ taking the form $s^{*}(X(\cdot))=s^{* *}\left(\sum_{i=1}^{N} X_{i}(\cdot)\right)$. Indeed $s^{*}(\cdot)$ has the form

$$
s^{*}(X)=w+\gamma(E)+\gamma^{\prime}(E)\left(\sum_{i=1}^{N} X_{i}(1)\right)+\frac{r}{2} \gamma^{\prime}(E)^{2} \sigma^{2}
$$

where $\sigma^{2}=\sum_{i=1}^{N} \sum_{j=1}^{N} \sigma_{i j}$.

Proof: The first-order conditions for $\mu(E)$ can be written as:

$$
\hat{c}_{i}(\mu(E)) \Leftrightarrow u_{i}+v_{i}=\lambda, \quad i=1, \ldots, N,
$$

where $u_{i}, v_{i}, i=1, \ldots, N$, are nonnegative, with $u_{i}\left(\mu_{i} \Leftrightarrow \underline{m}_{i}\right)=v_{i}\left(M_{i} \Leftrightarrow \mu_{i}\right)=0$, and $\lambda$ is the Lagrange multiplier of the constraint $\sum_{i=1}^{N} \mu_{i}=E$. By the envelope theorem, one also has

$$
\gamma^{\prime}(E)=\lambda
$$

The lemma now follows directly from Proposition C.1, Remark C.1, and Corollary C.1. Q.E.D. 
Proposition C.2 Suppose that $E^{*}$ maximizes the expression

$$
E \Leftrightarrow \gamma(E) \Leftrightarrow \frac{r}{2} \gamma^{\prime}(E)^{2}\left[\sum_{i=1}^{N} \hat{p}_{i} k_{i}^{2} \Leftrightarrow \sum_{i=1}^{N} \sum_{j=1}^{N} \hat{p}_{i} \hat{p}_{j} k_{i} k_{j}\right]
$$

over the interval $\left[\sum_{i=1}^{N} \underline{m}_{i}, \sum_{i=1}^{N} M_{i}\right]$. If the principal observes the aggregate process $Y(\cdot):=\sum_{i=1}^{N} X_{i}(\cdot)$, but not its individual components, then a solution to the principal's problem is given by the control process $\mu^{* *}(\cdot)$ satisfying $\mu^{* *}(t)=\mu\left(E^{*}\right)$ for all $t$, implemented by the incentive scheme $s^{*}(\cdot)$ given as

$$
s^{*}(X)=w+\gamma\left(E^{*}\right)+\gamma^{\prime}\left(E^{*}\right)\left(\sum_{i=1}^{N} X_{i}(1)\right)+\frac{r}{2} \gamma^{\prime}\left(E^{*}\right)^{2} \sigma^{2} .
$$

Proof: If the principal observes only the aggregate outcome process, any incentive scheme $s^{*}(\cdot)$ that he uses must take the form $s^{*}(X(\cdot))=s^{* *}\left(\sum_{i=1}^{N} X_{i}(\cdot)\right)$. With such an incentive scheme, by Corollary C.1, he can implement a control process $\mu^{*}(\cdot)$ if and only if there exist adapted processes $u_{i}(\cdot), v_{i}(\cdot), i=1, \ldots, N$, and $\lambda(\cdot)$, such that for all $i, u_{i}(\cdot)$ and $v_{i}(\cdot)$ take nonnegative values, and for almost all $t$, with probability one, $\mu^{*}(t)$ minimizes (C.1) over the set $\prod_{i=1}^{N}\left[\underline{m}_{i}, M_{i}\right]$, and moreover $\hat{c}_{i}\left(\mu^{*}(t)\right) \Leftrightarrow u_{i}(t)+v_{i}(t)=\lambda(t)$ for $i=1, \ldots, N$. Since $\mu^{*}(t)$ minimizes (C.1) over the set $\prod_{i=1}^{N}\left[\underline{m}_{i}, M_{i}\right]$, one also has $u_{i}(t)\left(\mu_{i}^{*}(t) \Leftrightarrow \underline{m}_{i}\right)=$ $v_{i}(t)\left(M_{i} \Leftrightarrow \mu_{i}^{*}(t)\right)=0$. Thus $\mu^{*}(t)$ satisfies the first-order conditions for the problem of minimizing $\hat{c}(\mu)$ over the set $\prod_{i=1}^{N}\left[\underline{m}_{i}, M_{i}\right]$ under the constraint that $\sum_{i=1}^{N} \mu_{i}(t)=E(t)$, where $E(t):=\sum_{i=1}^{N} \mu_{i}^{*}(t)$. Given that $\hat{c}(\cdot)$ is convex, it follows that for almost all $t$, with probability one, $\mu^{*}(t)=\mu(E(t)), \hat{c}\left(\mu^{*}(t)\right)=\gamma(E(t))$, and $\hat{c}_{i}\left(\mu^{*}(t)\right) \Leftrightarrow u_{i}(t)+v_{i}(t)=$ $\gamma^{\prime}(E(t))$. Upon using these equation to substitute in (C.2), we find that the function $s^{* *}(\cdot)$ has the representation

$$
s^{* *}(Y)=w+\int_{0}^{1} \gamma(E(t)) d t+\int_{0}^{1} \gamma^{\prime}(E(t)) d Y(t)+\frac{r}{2} \int_{0}^{1} \gamma^{\prime}(E(t))^{2} d t \sigma^{2} .
$$

Conditional on the process $E(\cdot)$, the principal's net expected payoff is then almost surely equal to

$$
\int_{0}^{1} E(t) d t \Leftrightarrow w \Leftrightarrow \gamma(E(t)) d t \Leftrightarrow \frac{r}{2} \int_{0}^{1} \gamma^{\prime}(E(t))^{2} d t \sigma^{2} .
$$

The definition of $E^{*}$ implies that with probability one this is no larger than

$$
E^{*} \Leftrightarrow w \Leftrightarrow \gamma\left(E^{*}\right) \Leftrightarrow \frac{r}{2} \gamma^{\prime}\left(E^{*}\right)^{2} \sigma^{2}
$$


Upon taking expectations with respect to the process $E(t)$, one immediately sees that the principal's net expected payoff from any control process $\mu^{*}(\cdot)$ which is implementable by an incentive scheme of the form $s^{*}(X(\cdot))=s^{* *}\left(\sum_{i=1}^{N} X_{i}(\cdot)\right)$ cannot exceed his net expected payoff from the control process $\mu^{* *}(\cdot)$ satisfying $\mu^{* *}(t)=\mu\left(E^{*}\right)$ for all $t$. Under the given information assumption the control process $\mu^{* *}(\cdot)$ is therefore indeed optimal for the principal. The representation (C.9) of the incentive scheme that implements $\mu^{* *}(\cdot)$ is obtained by substituting for $E(t)=E^{*}$ in (C.10) and using the fact that $\int_{0}^{1} d Y(t)=$ $Y(1) \Leftrightarrow Y(0)=\sum_{i=1}^{N} X_{i}(1) \Leftrightarrow \sum_{i=1}^{N} X_{i}(0)=\sum_{i=1}^{N} X_{i}(1)$.

Q.E.D. 


\section{REFERENCES}

Berge, C. (1959): Espaces Topologiques. Paris: Dunod.

Billingsley, P. (1968): Convergence of Probability Measures. New York: John Wiley \& Sons.

Bolton, P., And C. Harris (1997): "The Continuous-Time Principal-Agent Problem: First-Best Risk-Sharing Contracts and their Decentralization," mimeo, King's College, Cambridge.

Ginman, I., And A. W. Skorokhod (1979): Theory of Stochastic Processes, Vol. III. Heidelberg: Springer.

Hellwig, M. (1998a): "Aggregation and Linearity in Multi-Period Mean-Variance Incentive Problems," mimeo, University of Mannheim.

_ (1998b): "A Maximum Theorem for the Transition between Discrete- and Continuous-Time Intertemporal Agency Problems à la Holmström and Milgrom," mimeo, University of Mannheim.

Holmström, B., AND P. Milgrom (1987): "Aggregation and Linearity in the Provision of Intertemporal Incentives," Econometrica, 55, 303-328.

(1991): "Multi-Task Principal Agent Analyses," Journal of Law Economics, and Organization, 7 (Sp.), 24-52.

Ітон, Н. (1992): "Cooperation in Hierarchical Organizations: An Incentive Perspective," Journal of Law, Economics and Organization, 8, pp. 321-345.

Mirrlees, J. (1974): "Notes on Welfare Economics, Information and Uncertainty," in: Essays on Economic Behavior Under Uncertainty, ed. by M. Balch, D. McFadden and S. Wu. Amsterdam: North-Holland, 243-58.

Müller, H. (1997): "Randomization in Dynamic Principal-Agent Problems," Discussion Paper No. 97-37, SFB 504, University of Mannheim.

Schättler, H., And J. Sung (1993): "The First-Order Approach to the ContinuousTime Principal-Agent Problem with Exponential Utility," Journal of Economic Theo- 
ry, 61, 331-371.

(1997): "On Optimal Sharing Rules in Discrete- and Continuous-Time PrincipalAgent Problems with Exponential Utility," Journal of Economic Dynamics and Control, 21, 551-574.

Sung, J. (1995): "Linearity with Project Selection and Controllable Diffusion Rate in Continuous-Time Principal-Agent Problems," RAND Journal of Economics, 26, 720743.

(1997): "Corporate Insurance and Managerial Incentives," Journal of Economic Theory, 74, 297-332. 